\title{
Distinct Modulations in Sensorimotor Postmovement and Foreperiod $\beta$-Band Activities Related to Error Salience Processing and Sensorimotor Adaptation
}

\author{
Flavie Torrecillos, Julie Alayrangues, Bjørg Elisabeth Kilavik, and Nicole Malfait \\ Institut de Neurosciences de la Timone, UMR7289, CNRS/Aix Marseille Université, 13005 Marseille, France
}

In a recent study, Tan et al. (2014a,b) showed that the increase in $\beta$-power typically observed after a movement above sensorimotor regions ( $\beta$-rebound) is attenuated when movement-execution errors are induced by visual perturbations. Moreover, akin to sensorimotor adaptation, the effect depended on the context in which the errors are experienced. Thus the $\beta$-rebound attenuation might relate to neural processes involved in trial-to-trial adaptive mechanisms. In two EEG experiments with human participants, along with the $\beta$-rebound, we examine $\beta$-activity during the preparation of reaches immediately following perturbed movements. In the first experiment, we show that both foreperiod and postmovement $\beta$-activities are parametrically modulated by the sizes of kinematic errors produced by unpredictable mechanical perturbations (force field) independent of their on-line corrections. In the second experiment, we contrast two types of reach errors: movement-execution errors that trigger trial-to-trial adaptive mechanisms and goal errors that do not elicit sensorimotor adaptation. Movement-execution errors were induced by mechanical or visual perturbations, whereas goal errors were caused by unexpected displacements of the target at movement initiation. Interestingly, foreperiod and postmovement $\beta$-activities exhibit contrasting patterns, pointing to important functional differences of their underlying neuronal activity. While both types of reach errors attenuate the postmovement $\beta$-rebound, only the kinematic errors that trigger trial-to-trial motor-command updates influenced $\beta$-activity during the foreperiod. These findings suggest that the error-related modulation of the $\beta$-rebound may reflect salience processing, independent of sensorimotor adaptation. In contrast, modulations in the foreperiod $\beta$-power might relate to the motorcommand adjustments activated after movement-execution errors are experienced.

Key words: arm movements; $\beta$-band oscillations; EEG; error processing; force field; sensorimotor adaptation

Significance Statement

The functional significance of sensorimotor $\beta$-band $(15-25 \mathrm{~Hz})$ oscillations remains uncertain. Recently $\beta$-power was found to be reduced following erroneous movements. We extend and refine this novel finding in two crucial ways. First, by contrasting the EEG correlates of movement errors driving or not driving adaptation we dissociate error-salience processing from error-based adaptation. Second, in addition to $\beta$-activity in error trials, we examine $\beta$-power during the preparation of the subsequent movements. We find clearly distinct patterns of error-related modulations for $\beta$-activities preceding and succeeding movements, highlighting critical functional differences. Postmovement $\beta$-power may reflect error-salience processing independent of sensorimotor adaptation. In contrast, modulations in the foreperiod $\beta$-band power may directly relate to the motor-command adjustments activated after movement-execution errors are experienced.

\section{Introduction}

While oscillations are omnipresent in the brain, it is still disputed whether they serve specific functions. $\beta$-band $(15-25 \mathrm{~Hz})$ oscil-

Received March 20, 2015; revised Aug. 7, 2015; accepted Aug. 9, 2015.

Author contributions: F.T. and N.M. designed research; F.T., J.A., and N.M. performed research; F.T., J.A., B.E.K., and N.M. analyzed data; F.T., J.A., B.E.K., and N.M. wrote the paper.

This research was supported by the Agence Nationale pour la Recherche and the European Union H2020 MSCAITN program. We thank Andrea Brovelli, Bill Mackay, and Manuel Zaepffel for helpful discussions, as well as two anonymous reviewers for crucial comments and suggestions.

The authors declare no competing financial interests. lations often dominate in sensorimotor cortex, but their functional roles remain uncertain (for review, see Kilavik et al., 2013). Prominent at rest, they have been originally attributed to an "idling" state of the motor system (Jasper and Penfield, 1949; Pfurtscheller et al., 1996). In line with this, $\beta$-oscillations within

Correspondence should be addressed to Nicole Malfait, Institut de Neurosciences de la Timone (INT), UMR7289 CNRS—Aix Marseille Université, Campus santé Timone, 27, boulevard Jean Moulin, 13385 Marseille cedex 5. E-mail: nmalfait@gmail.com.

DOI:10.1523/JNEUROSCI.1090-15.2015

Copyright $\odot 2015$ the authors $\quad 0270-6474 / 15 / 3512753-13 \$ 15.00 / 0$ 
and beyond sensorimotor cortex were recently proposed as an active process that promotes the existing motor (or cognitive) state and compromises neuronal processing of new movement (or cognitive) states (Gilbertson et al., 2005; Engel and Fries, 2010). However, increased interareal synchrony in the $\beta$-range was also linked to top-down control (Buschman and Miller, 2007; Siegel et al., 2012) and heightened visuomotor attention (Roelfsema et al., 1997; Classen et al., 1998), suggesting more general roles for $\beta$-oscillations in the flow of information.

Recently, Tan et al. (2014a,b) examined sensorimotor $\beta$-band activity during a joystick task in which visual perturbations were introduced. They found that the $\beta$-rebound, an increase in $\beta$-power typically observed at the end of a movement, was attenuated in trials with movement-execution errors. Furthermore, this effect was stronger when contextual information enhanced the behavioral salience of the kinematic errors. In a similar way, trial-to-trial motor-command adjustment is not constant as a function of error magnitude and is influenced by the task relevance of error and its sensory uncertainty (Korenberg and Ghahramani, 2002; Fine and Thoroughman, 2007; Burge et al., 2008; van Beers, 2009; Wei and Körding, 2009; Marko et al., 2012; Sober and Brainard, 2012), suggesting that the attenuation of the $\beta$-rebound, following movement errors, might be related to neural processes involved in sensorimotor adaptation. This is in line with the proposition that attenuations of the $\beta$-power facilitate changes in the motor set (Engel and Fries, 2010).

To further investigate the functional significance of the $\beta$-rebound attenuation, we dissociated error salience from trialto-trial adaptation. In two complementary experiments, we studied modulations of the postmovement $\beta$-rebound by different types of reach errors. As adaptive motor-command adjustments might be reflected in the preparation of subsequent movements, we also examined $\beta$-band activity during the preparation of reaches immediately after perturbed movements. Some studies have suggested a link between $\beta$-oscillations and motor planning in the foreperiod, between the warning and imperative stimuli (Alegre et al., 2006; Zaepffel et al., 2013; for review, see Kilavik et al., 2013). In the first experiment, participants reached to a visual target in a force field created by a robotic device, and movementexecution errors of different sizes were produced by unpredictable changes in the strength of the applied force (Torrecillos et al., 2014). In the second experiment, we contrasted two types of reach errors: movement-execution errors that trigger trial-to-trial changes in motor commands and goal errors that do not elicit sensorimotor adaptation. In catch trials, movement-execution errors were induced by mechanical or visual perturbations, whereas goal errors were caused by unexpected displacements of the target upon movement initiation (Diedrichsen et al., 2005).

Consistent with Tan et al. (2014a), we observed that the postmovement $\beta$-rebound was parametrically attenuated by the sizes of movement-execution errors produced by mechanical perturbations. Furthermore, the $\beta$-rebound was modulated by reach errors independently of their correction on-line (Experiment 1). Importantly, this error-related attenuation of the postmovement $\beta$-rebound appeared to be insensitive to the nature of the reach errors, as it was present for both movement-execution and goal errors (Experiment 2). Interestingly, the premovement foreperiod $\beta$-activity presented a clearly contrasting pattern, because it was modulated only for movements that followed kinematic errors driving trial-to-trial motor-command updates. Our findings suggest that the error-related modulation of the postmovement $\beta$-rebound may reflect salience processing independent of sensorimotor adaptation. In contrast, modulations in the foreperiod $\beta$-band power might be directly related to the motor-command adjustments activated after movement-execution errors are experienced.

\section{Materials and Methods}

In Experiment 1, our objective was to produce movement-execution errors of different amplitudes. To dissociate reach errors from on-line motor corrections, we took advantage of a "shooting" task with ballistic movements in which participants experience their errors without having the opportunity to correct them within the same trial (Tseng et al., 2007). In Experiment 2 (including two experimental protocols), our aim was to dissociate reach errors from sensorimotor adaptation. For this purpose, we used a pointing task (allowing for on-line motor correction) in which we induced reach errors of two different types: movement-execution errors that triggered adaptive processes and goal errors that did not result in sensorimotor remapping (Diedrichsen et al., 2005). Movementexecution errors were induced by mechanical (force field) or visual (rotation) perturbations, whereas goal errors were induced by unexpectedly displacing the target upon movement initiation. Note that, in contrast to target jumps that occur during a saccade (Goodale et al., 1986; Prablanc and Martin, 1992), no trial-to-trial adaptation is induced in this case.

\section{Participants}

A total of 35 healthy males aged 24.7 years (range 18-35 years) took part in the study. Fifteen volunteers participated in Experiment 1. Fourteen and 10 volunteers participated in two different protocols of Experiment 2 , respectively. One volunteer participated in all three experiments and two participated in both protocols of Experiment 2. Except one ambidextrous individual (who participated in Experiment 1), all participants were right-handed, as assessed by the Edinburgh Handedness Inventory (Oldfield, 1971) and all had normal or corrected-to-normal vision. All participants were free of known neurological or psychiatric disorders and gave informed consent according to a protocol approved by the Ethics Board of the Institut de Neurosciences de la Timone.

\section{Experimental setup}

All experiments were performed using a robotic exoskeleton (KINARM; BKIN Technologies) that allows flexion and extension movements of the elbow and shoulder in the horizontal plane and that can apply mechanical loads to each of these two joints (Fig. $1 A$ ). Participants were seated with their right arm installed in the exoskeleton; the height of the chair was adjusted so that the shoulder was abducted by $\sim 70^{\circ}$. Using a semisilvered mirror, direct vision of the hand was prevented throughout the task while a cursor representing the index fingertip and the visual display was projected onto the same plane as the (not visible) hand. Participants maintained their right hand in a pointing position. Head movements were limited by using a chin rest.

\section{Task: Experiment 1}

The task and the experimental protocol of the first experiment has been described in detail in a previous study (Torrecillos et al., 2014) that presented other results on EEG signals recorded during the same experiment.

Participants performed a "shooting" task in which they were instructed to reach straight ahead with no on-line movement corrections (Tseng et al., 2007). Movements were always initiated from the same start circle $(1.5 \mathrm{~cm}$ diameter) located at the center of an outer ring $(10 \mathrm{~cm}$ radius). In initial position, the elbow joint was anteflexed by $90^{\circ}$ and the shoulder horizontally abducted by $45^{\circ}$. Participants were instructed to reach in the direction of a small white donut $(0.5 \mathrm{~cm}$ diameter $)$ located 5 $\mathrm{cm}$ away and to move out of the outer ring within $375 \pm 50 \mathrm{~ms}$ (Fig. 1B).

To initiate a trial, participants had to maintain their index finger in the start circle for $500 \mathrm{~ms}$ before it disappeared to warn them to get ready (READY). Following a $1500 \mathrm{~ms}$ delay, the donut turned into a white disk to indicate that the movement could be initiated (GO). Participants were instructed to keep their hand stationary during this fixed foreperiod. Importantly, they were also clearly informed that they were not performing a reaction-time task and should take all the time they needed to plan movements executed within the prescribed time window. 


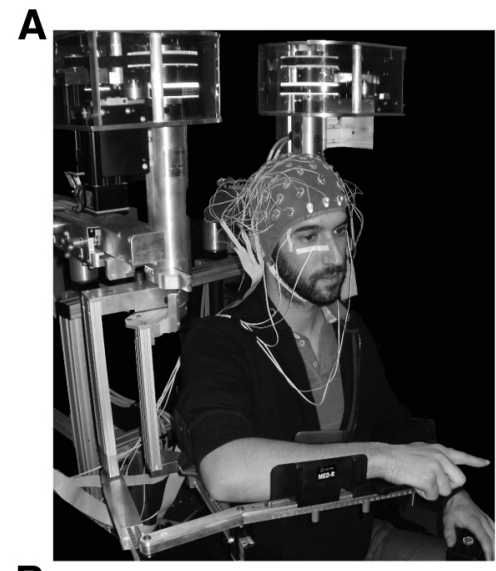

B

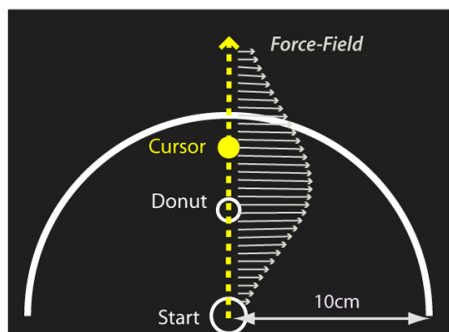

Exp 1: Shooting task
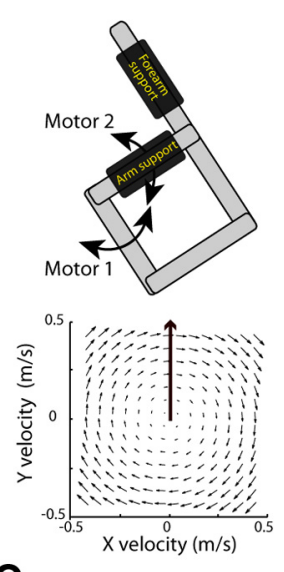

C

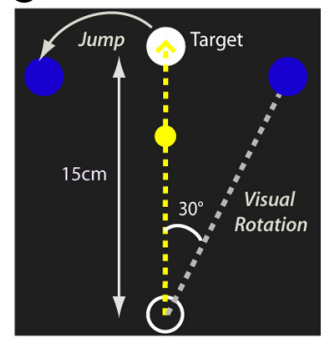

Exp 2: Pointing task

Figure 1. Experimental setup. $\boldsymbol{A}$, For both experiments, EEG was recorded from participants performing reaching movements while installed in a robotic exoskeleton (left) that can apply mechanical loads to the shoulder and elbow joints through two torque motors (top right). In force-field catch trials, the robot was programmed to create a viscous curl field in which forces were proportional and acted perpendicularly to the velocity of the hand (bottom right). $\boldsymbol{B}$, In Experiment 1, participants performed a "shooting" task in which they were instructed to move in the direction of a small donut without stopping, and to end their movements $\sim 3-4 \mathrm{~cm}$ beyond an outer ring. Mechanical perturbations were produced by unexpected changes in the amplitude of the force field (arrows). C, In Experiment 2, participants performed a pointing task in which they were instructed to reach toward and stop their movements in a visual target. Throughout the task, three possible target locations were indicated in dark blue. In all trials, the central target was turned on (white). In force-field catch trials, the force field was unexpectedly activated. In visual rotation catch trials, the cursor representing the index fingertip of the participant was rotated by $30^{\circ}$ clockwise relative to the actual hand position. In the target-jump catch trials the target moved to the leftward location $\left(30^{\circ}\right)$ upon movement onset.

Movement duration was computed on-line and corresponded to the delay between the time when hand speed exceeded $7 \mathrm{~cm} / \mathrm{s}$ and the time at which the fingertip cursor reached the outer ring. Participants received a visual feedback about the duration of their movement at the time the cursor reached the outer ring; the white disk turned back into a donut; it was colored green, red, or white to indicate that the movement was too slow, too fast, or performed at the correct speed, respectively. In perturbed trials (see below) it turned back into a white donut regardless of movement duration.

To reduce final movement correction, the fingertip cursor visible during the movement disappeared upon reaching the outer ring. After a delay of $500 \mathrm{~ms}$, the arm of the participant was passively brought back in its initial configuration. No visual hand feedback was provided during the passive return that lasted $1500 \mathrm{~ms}$. The fingertip cursor and the start circle reappeared only when the hand was back in its initial location. Each trial lasted $\sim 5 \mathrm{~s}$ throughout which the participants were required to keep fixating the small donut/disk and to avoid eye blinks and saccades.

\section{Experimental protocol: Experiment 1}

Kinematic errors of different sizes were induced by mechanical perturbations of different amplitudes, with the aim to study their EEG correlates. The robotic device was programmed to create a clockwise curl field in which the force is proportional and acts perpendicular to the velocity of the hand. The force was set to $\mathbf{f}=\mathbf{B} * \mathbf{v}$, where $\mathbf{f}=[\mathrm{fx}, \mathrm{fy}]$ depends on the velocity of the

hand $\mathbf{v}=[\mathrm{vx}, \mathrm{vy}]$, and $\mathbf{B}=[0,-\alpha, \alpha, 0] \mathrm{N} * \sec * \mathrm{~m}^{-1}$, with $\alpha$ the force-amplitude parameter. The field was produced by the torque motors of the robotic exoskeleton, using the transformation from endpoint force to joint torque: $t=\mathrm{J}^{\prime} * \mathrm{f}$, where $\mathrm{J}=[11 * \sin \theta 1+12 * \sin (\theta 1+\theta 2), 12 * \sin (\theta 1$ $+\theta 2) ; 11 * \cos \theta 1+12 * \cos (\theta 1+\theta 2), 12 * \cos (\theta 1+\theta 2)]$ is the configurationdependent differential transformation matrix (Jacobian matrix). Four different force-field amplitudes were used by setting $\alpha=0,3,6$, or 9 . Handpath deviations were produced by unexpected changes in the amplitude of the force field.

The experiment included two phases: a preliminary phase, in which participants first learned the task while the motors of the robot remained off, and then adapted to the activated force field, and an experimental phase, in which $20 \%$ of the trials were perturbed by unexpectedly changing the force amplitude. EEG signals were recorded during the experimental phase only.

Preliminary phase (familiarization and force-field adaptation). After the calibration of the robotic device and verbal instructions about the task requirements, participants learned the shooting task, performing three blocks of 30 reaching movements with the motors of the robot turned off $(\alpha=0)$. During a fourth block of 30 trials, the force field was activated $(\alpha=9)$. Clearly deviated upon initial exposure to the force field, movements rapidly (within 10-15 trials) recovered their usual kinematics as participants adapted to the new force condition.

Experimental phase. The experimental phase consisted of 10 blocks of 120 trials each, including 96 trials (80\%) performed in the strongest force field $(\alpha=9)$ interspersed with $3 * 8$ catch trials $(20 \%)$ in which the amplitude of the force field was unpredictably reduced to $\alpha=6,3$, or 0 . Since participants had adapted to the strongest force field $(\alpha=9)$ during the preliminary phase, movements performed in this field were not perturbed and presented no systematic hand-path deviations. In contrast, reduced force-field catch trials, with $\alpha=6,3$, or 0 , were effectively perturbed and usually presented small, medium, and large hand-path deviations, respectively. To maintain adaptation to the strong force field $(\alpha=9)$, two catch trials were always separated by at least three unperturbed ( $\alpha=9$ ) trials. Each block lasted $\sim 10$ min and breaks of $\sim 2$ min were allocated between blocks, for total task duration of $\sim 2 \mathrm{~h}$.

\section{Task: Experiment 2}

A pointing task in which participants were allowed to correct their movements was used in the second experiment. Participants were instructed to reach and stop their movements in a visual target $(1.5 \mathrm{~cm}$ diameter) located $15 \mathrm{~cm}$ from the start circle $(1.5 \mathrm{~cm}$ diameter; Fig. $1 C)$. In the initial start position, the elbow joint was anteflexed $20^{\circ}$ and the shoulder horizontally abducted $110^{\circ}$. Throughout the experiment, three possible targets $\left(-30^{\circ}, 0,30^{\circ}\right)$ were indicated as dark-blue disks $(1.5 \mathrm{~cm}$ diameter). In each trial, the central straight-ahead target first turned into a white circle (READY) and then a white disk (GO), with a fixed foreperiod between READY and GO of 1500 ms during which participants maintained their hand stationary. In all trials, participants had to reach to this central target, except for target-jumps trials, in which upon movement initiation the central target was turned off at the same time as the target located to the left was turned on. Participants had to reach to the target within $375 \pm 25 \mathrm{~ms}$, and were provided feedback about the duration of their movements by a color change of the target disk: green, red, or dark blue to indicate that the movement was too slow, too fast, or performed at the correct speed, respectively. Movement duration was computed from movement onset (hand speed exceeding $7 \mathrm{~cm} / \mathrm{s}$ ) to the time when the index finger reached the target.

Trials unfolded as in Experiment 1, apart from two modifications introduced to limit temporal overlap between $\beta$-components reflecting distinct neural processes. In Protocol 1, the delay between the visual feedback, provided when the hand arrived at the target, and the start of the passive return to the initial hand location was increased from 500 to $1000 \mathrm{~ms}$. In Protocol 2, in addition we increased the delay between the end of the passive return and the READY cue of the subsequent trial from 500 to $2000 \mathrm{~ms}$, throughout which participants had to maintain their index finger in the start circle. Trials lasted $\sim 5.5$ $\mathrm{s}$ or $\sim 7 \mathrm{~s}$ during which participants were asked to keep fixating the central target location, except in the trials where the target was unex- 
pectedly displaced upon movement initiation, in which they were allowed to saccade to the new target location.

\section{Experimental protocols: Experiment 2}

In Protocol 1, EEG correlates of two different types of reach errors were examined. Movement-execution errors were induced by mechanical or visual perturbations, and goal errors were produced by unexpected displacements of the target after movement onset. Mechanical perturbations were applied using the same force field as in Experiment 1. However, participants did not adapt to the force field in a preliminary phase, and hand trajectories were perturbed by unexpectedly activating (not reducing) the force. Visual perturbations consisted in unexpected $30^{\circ}$ clockwise rotations (about the starting location) of the fingertip cursor from the actual finger position. Goal errors were produced by unexpected displacements of the target, from the central to the left location, upon movement initiation. In Protocol 2, we only induced movementexecution errors using the mechanical perturbations (force field). (Including all three perturbation conditions would have resulted in an excessively long experimental session.) EEG signals were recorded during the experimental phase only.

Preliminary phase (familiarization). As in the previous experiment, in both protocols after the calibration of the robot and the verbal instructions, participants learned the task performing two blocks of 30 reaching movements each. While all trials of the first block were performed with the motors off, four force-field catch trials were introduced in the second block, to evaluate the amplitude of the reach errors produced by the force field (the effect of a new force field varies with limb stiffness). The forceamplitude parameter was set to $\alpha=6$ or 7, for 10 and four participants, respectively, depending on the size of the hand-path deviations that were observed.

Experimental phase. In Protocol 1, as in Experiment 1, the experimental phase consisted of 10 blocks of 120 trials each, including 96 unperturbed trials $(80 \%)$ interspersed with $3 * 8$ catch trials $(20 \%)$ in which reach errors were produced: eight force-field and eight visual rotation catch trials in which movement-execution errors were produced, and eight target-jump catch-trials provoking goal errors. Two catch trials were always separated by at least three unperturbed trials. Each block lasted $\sim 11 \mathrm{~min}$ and breaks of $\sim 2$ min were allocated between blocks, with a task lasting $\sim 2 \mathrm{~h} 15 \mathrm{~min}$.

In Protocol 2, the experimental phase was shorter, and consisted of eight blocks of 60 trials each, including 48 unperturbed trials (80\%) interspersed with 12 catch trials $(20 \%)$ in which movement-execution errors were produced by unexpectedly activating the force field. Two catch trials were always separated by at least three unperturbed trials. Each block lasted $\sim 8 \mathrm{~min}$ and breaks of $\sim 2 \mathrm{~min}$ were allocated between blocks, for a task lasting $\sim 1$ h 20 min total.

\section{Behavioral data recording and analysis}

Identical recording and analysis techniques were used in all experiments. The angular position and velocity data of the motor resolvers were collected at $1000 \mathrm{~Hz}$. These signals were downsampled to $100 \mathrm{~Hz}$ off-line and then filtered with a second-order zero-phase shift, low-pass Butterworth filter (cutoff frequency of $10 \mathrm{~Hz}$ ). Finger position and velocity were calculated from these angular data. Kinematic data were analyzed using custom routines written in MATLAB (The MathWorks). Movement onset was defined as the time when the tangential velocity of the hand exceeded $7 \mathrm{~cm} / \mathrm{s}$ and movement offset as the time when tangential velocity and acceleration fell below the thresholds of 2.5 and $5 \mathrm{~cm} / \mathrm{s}^{2}$, respectively. The trials in which the hand was not maintained stable enough (tangential velocity $>5 \mathrm{~cm} / \mathrm{s}$ ) in the start position during the delay between the READY and GO signals, or in which the movement was initiated before the GO signal, were excluded from the analyses ( $\sim 8 \%$ of trials). Movement durations were defined as the delays between movement onset and movement offset. Reaction times were computed between the GO signal and movement onset.

For all experiments, we considered two subsets of the recorded data. The first subset was used to assess the impact of reach error on the postmovement $\beta$-rebound in perturbed trials. It included all the catch trials $n$ (three sets of 80 trials), plus 80 trials pseudorandomly selected among the 240 unperturbed $n-1$ movements that were used as baseline. (These latter trials offered the warranty of not having been influenced by a reach error produced in the preceding trial.) For Experiment 1, we defined four trial categories according to the observed hand-path deviations: No, Small, Medium or Large (Torrecillos et al., 2014). In Experiment 2, for Protocol 1, trials were divided into four categories depending on the applied perturbation: No, Force Field (FF), Visual Rotation (VR), or Target Jump (TJ). For Protocol 2, there were only the two trial categories No, FF.

The second data subset was used to determine whether error-related modulations could be observed during the preparation of movements performed just after a perturbed trial. It included all the reaches $n+1$ that directly followed a deviated movement $n$, which were categorized according to the trial they succeeded: after-Small, after-Medium, or after-Large, for Experiment 1; after-FF, after-VR, or after-TJ; for the first protocol of Experiment 2; and after-FF for the second protocol. In all cases, the No trials defined above were also here used as baseline.

In Experiment 1, kinematic errors were quantified by the hand-path deviations perpendicular to the straight line connecting the start position and the donut, measured at peak tangential velocity (PD-vel). In Experiment 2, we completed this measure with another one: the hand-path length (HPL) defined as the length of the path covered by the hand between the GO signal and the moment when presenting the movementduration feedback. This second measure allowed capturing of the final corrective movements present in the pointing task.

Statistical tests were run using the free software R. For Experiment 1, a repeated-measures ANOVA was run on the PD-vel. For Experiment 2, a repeated-measures MANOVA (Protocol 1) or a paired Hotelling's T-square test (Protocol 2) was run on the two measures PD-vel and HPL. In all cases, trial category was used as within-subject factor. Normality and sphericity assumptions were controlled and Huyn-Feldt correction applied whenever appropriate. For all tests, the significance threshold was set to 0.05 and multiple-comparison corrections were performed with the Bonferroni procedure.

\section{EEG recording}

EEG activity was recorded continuously at $1024 \mathrm{~Hz}$ using a 64-channel BioSemi ActiveTwo system (BioSemi) referenced to the Common Mode Sense-Drive Right Leg ground. Electrodes were embedded into an elastic cap and evenly distributed over the scalp according to the extended 10-20 EEG system. EOG activity was recorded with surface electrodes placed near both outer canthi (saccades) as well as under and above the left orbit (blinks).

\section{EEG preprocessing}

EEG data preprocessing was performed using the free software ELAN (http://elan.lyon.inserm.fr/; Aguera et al., 2011). Continuous signals were bandpass filtered between 0.5 and $100 \mathrm{~Hz}$ (Butterworth order 2). Changes in signal amplitude exceeding $100 \mu \mathrm{V} / 500 \mathrm{~ms}$ were automatically detected. Data were then visually inspected for any remaining nonstereotyped artifacts; corresponding channels and signal segments were excluded from further analysis. Stereotypical artifacts such as blinks, eye movements, or muscular activity were removed by an Independent Component Analysis procedure (Delorme et al., 2007). The data were once more visually inspected for remaining artifacts. The resulting EEG data were then converted to current source density to increase spatial selectivity and minimize volume conduction.

\section{EEG time-frequency analysis}

Time-frequency analyses were performed using the free software ELAN (http://elan.lyon.inserm.fr/; Aguera et al., 2011) and custom-written MATLAB routines (The MathWorks). Each single trial signal was transformed in the time-frequency domain by convolution with the complex Morlet wavelets characterized by the ratio $f 0 / \sigma f=7$, with $f 0$ ranging from 13 to $50 \mathrm{~Hz}$ in $0.5 \mathrm{~Hz}$ steps. To estimate event-related $\beta$-power changes we normalized the data with respect to the average power computed across all trials, as no clear baseline could be defined during the task (see Tan et al., 2014a, b for a similar procedure). Before normalization, the raw power data were log transformed. Then, the log-power at each frequency and each time point was normalized relative to the average log- 


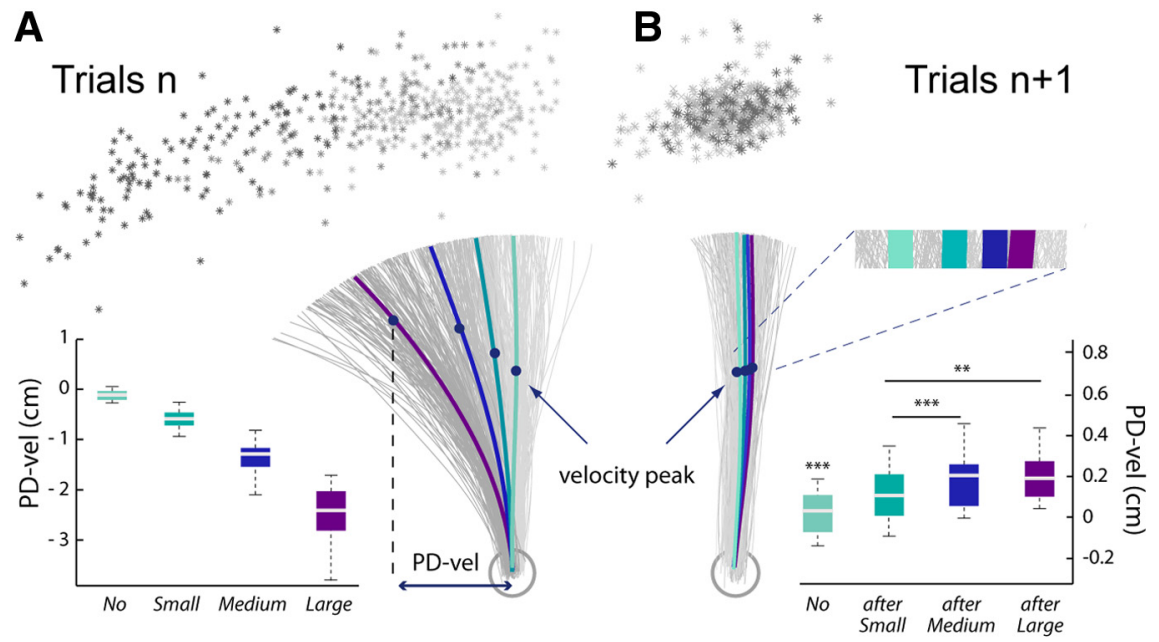

Figure 2. Behavioral data from the shooting task (Experiment 1). $A$, In Experiment 1, the mechanical perturbations applied in the catch trials $n$ produced Small, Medium, or Large kinematics errors. For a representative participant, hand paths for all individual trials of the experimental phase are plotted in gray; the stars indicate the hand locations at the end of the reaching movements. The mean hand paths for the different categories of trials ( $N$ o, Small, Medium, and Large) are plotted in thick lines. The values PD-vel observed at the group level $(n=15)$ are indicated for the four different categories of trials in the boxplots. $\boldsymbol{B}$, In addition to reach errors observed in the catch trial $n$, slight hand-path deviations in the opposite direction were visible in the subsequent trials $n+$ 1. For the same participant, individual and mean hand paths are plotted for the No, after-Small, after-Medium, and after-Large trials. Hand positions at velocity peaks and movement ends are also indicated. Group data of the PD-vel measures and statistical results are indicated for the different categories of trials in the boxplots.

power of that frequency previously computed over all the trials of the experimental session. For each participant and each time point, the mean power changes were averaged in a specific $\beta$-frequency band (see below) across all trials for each category and smoothed using a Gaussian Kernel with 250 sampling points $(244 \mathrm{~ms})$ full-width at half-maximum. For Experiment 1 , on average $74 \pm 2.2$ trials were used for each category to compute the individual power curves. For Experiment 2, on average $70.4 \pm 2.6$ and $80.2 \pm 2.4$ trials for each category were included in the analyses for Protocols 1 and 2, respectively.

In all experiments, we examined modulation of $\beta$-band activity during two distinct trial epochs of interest, defined relative to movement offset (trials $n$ ) and movement onset (trials $n+1$ ), respectively. They will be called "postmovement" and "premovement" epochs further in the text, for the sake of simplicity, even though they are both large enough to extend into the movement-execution period. To examine the errorrelated modulation of the $\beta$-rebound, we defined a postmovement window extending from -500 to +1500 ms around movement offset. To investigate the modulation of the $\beta$-band activity during reach preparation by an error that occurred in the previous trial, we considered a window covering -3000 to $500 \mathrm{~ms}$ around movement onset for Experiment 1 and the first protocol in Experiment 2, and from -4500 to $500 \mathrm{~ms}$ in Protocol 2.

Because of interindividual differences in head/brain anatomy and the exact electrode placements, the location of the most prominent eventrelated $\beta$-power changes differed substantially across participants. Thus considering the signals aligned to the offset (trials $n$ ) or the onset (trials $n$ +1 ) of the movement, for each participant, we selected the channel and the $10 \mathrm{~Hz}$ wide frequency band exhibiting the largest event-related $\beta$-power change in the unperturbed trials. (Note that for the second protocol in Experiment 2 the selection was slightly different we considered for each trial epoch of interest the largest $\beta$-power difference between the two categories of trials No and the FF or after-FF trials.) For each trial epoch of interest, group $\beta$-power profiles were obtained (for illustrative purposes) by averaging over the individually selected electrodes and frequency bands.

As a control, for Experiment 1 we repeated all statistical analyses (see below) using the same region of interest (C1-C3-CP1-CP3) and the same $18-28 \mathrm{~Hz}$ frequency band for all participants, and obtained similar results (not detailed here). Nevertheless, given the interindividual variabil- ity we chose to favor the procedure where frequency bands and electrodes were selected on an individual basis.

\section{EEG statistical analyses}

Statistical analyses were performed using the free software R and custom-written MATLAB routines (The MathWorks). First, the impact of reach errors on $\beta$-activity was assessed through mixed-effect linear models. Statistical analyses were computed over sliding windows of $50 \mathrm{~ms}$ with a time shift between successive windows of $10 \mathrm{~ms}$. Significance level was set to 0.05. The False Discovery Rate method was used to correct for multiple comparisons along the time axis, and the Bonferroni procedure was used to correct for multiple pairwise post hoc comparisons.

In Experiment 1, the parametric modulation of the $\beta$-activity was assessed by linear mixedeffects regressions across the four trial categories: No, Small, Medium, Large, in the postmovement epoch and No, after-Small, after-Medium, and after-Large in the premovement epoch. Specifically, we assessed whether $\beta$-power was attenuated in proportion to the sizes of the reach errors (the MAT$\mathrm{LAB}$ function fitlmematrix with the contrast $[3$ $1-1-3]$ was used).

In Experiment 2, for both the premovement and postmovement $\beta$-power activities, we first computed repeated-measures ANOVAs. Then we proceeded to pairwise comparisons (paired $t$ tests with Bonferroni correction) in which we compared each of the included perturbed conditions (TJ, FF, and VR, and after-TJ, after-FF, and after-VR) to the unperturbed trials No.

\section{Results}

In Experiment 1, first our aim was to determine whether the $\beta$-rebound was parametrically modulated by the sizes of kinematic errors provoked by mechanical perturbations, independently of their on-line corrections. Second, we wanted to assess whether the movement-execution error experienced in a catch trial impacted $\beta$-activity observed during the preparation of the movement in the subsequent trial. Experiment 2 was intended to investigate further the nature of the relationship between postmovement and premovement epoch $\beta$-activities and sensorimotor adaptation. For this purpose, we dissociated the size of the experienced reach error from the amplitude of the motor-command adjustment/adaptation observed in the subsequent trial. This was achieved by contrasting two types of reach errors: movement-execution errors that triggered adaptive mechanisms and goal errors that did not elicit sensorimotor adaptation (Diedrichsen et al., 2005).

\section{Experiment 1}

\section{Behavioral performance}

Kinematic errors of variable sizes were produced by unexpected changes in the amplitude of the applied force field. As expected, because of the nature of the shooting task (Tseng et al., 2007), no on-line movement correction was observed, as illustrated by the hand paths of a representative participant (Fig. $2 A$ ). There was no significant difference between the reaction times observed for the perturbed $n$, after $n+1$ and unperturbed No trials $\left(F_{(2,28)}=0.25\right.$, $p=0.77$; No: $525 \pm 24 \mathrm{~ms}$, perturbed: $520 \pm 23 \mathrm{~ms}$, after trials: $517 \pm 23 \mathrm{~ms}$ ), in contrast to movement durations that were significantly longer for perturbed than for No and after trials $\left(F_{(2,28)}=\right.$ 58.7, $p<0.001$; No: $585 \pm 12 \mathrm{~ms}$, perturbed: $641 \pm 10 \mathrm{~ms}$, after tri- 
als: $589 \pm 10 \mathrm{~ms}$; No vs perturbed: $t_{(14)}=-7.38$, after vs perturbed: $t_{(14)}=-10.25, p<0.001$ in all cases).

Kinematic errors were quantified by the hand-path perpendicular deviation measured at maximum velocity (PD-vel); group data for the different trial categories (No, Small, Medium, and Large) are also presented in Figure $2 A$. (Note that these PD-vel values are slightly different from those reported in Torrecillos et al. (2014) due to more severe trial-exclusion criteria in the present study.)

In addition to the clear reach errors observed in the perturbed catch trial $n$, slight hand-path deviations in the opposite direction were visible in the subsequent trials $n+1$. Figure $2 B$ shows individual hand paths for the same participant, along with group data. These deviations were proportional to the size of the error produced in the catch trial $n$ and in accordance with previous studies (Diedrichsen et al., 2005). A repeated-measures ANOVA on the PDvel revealed a significant effect of the trial category (no, after-Small, after-Medium, and after-Large; $\left.F_{(3,42)}=28.9, p<0.001\right)$. Post hoc comparisons revealed that the hand-path deviations were significantly larger for after-Small, after-Medium, and after-Large trials than for No trials (after-Small vs No: $t_{(14)}=5.69$, after-Medium vs No: $t_{(14)}=8.35$, after-Large vs No: $t_{(14)}=6.15 ; p<0.001$, in all cases). Also, deviations were larger for after-Medium and after-Large trials than for after-Small trials (after-Medium vs after-Small: $t_{(14)}=$ 7.33 and after-Large vs after-Small: $t_{(14)}=4.1, p<0.001$ and $p<$ 0.01 , respectively).

\section{EEG time-frequency data}

For illustrative purposes, in Figure $3 A$ we show on the same graph the general (group) time course of the tangential hand velocity and the $\beta$-power change observed for the unperturbed trials $n-1$. Both the EEG and kinematic profiles were obtained by aligning to the movement onset. The group velocity profile is the average of all individual mean profiles. The group $\beta$-power profile is the average over $18-28 \mathrm{~Hz}$ of the group time-frequency map (Fig. 3B). The map was obtained by averaging over the activity recorded at the electrodes individually selected based on their activity during the trial epoch defined relative to movement onset (premovement epoch) in unperturbed trials (see Materials and Methods). A large peak in $\beta$-power could be observed during the foreperiod (approximately -2000 to $-500 \mathrm{~ms}$ before movement onset; see reaction times above), centered around $1200 \mathrm{~ms}$ before movement onset. It was followed by a clear decrease in oscillatory power: the typical movement-related $\beta$ event-related desynchronization ( $\beta$-ERD) starting before movement onset and reaching a minimum around peak hand tangential velocity. A second $\beta$-power peak was visible at the end of movement, the postmovement $\beta$-rebound. (Note that in Figure 3 , the amplitude of the $\beta$-rebound is not optimally represented, since EEG signals are aligned to movement onset, not to movement offset.) Finally, the passive movement, when the robot brings the hand of the participant back to the starting position, induced a secondary decrease in oscillatory power (passive movementrelated $\beta$-ERD).

Postmovement $\beta$-rebound. Within the trial epoch defined relative to movement offset (postmovement epoch in trials $n$ ), $\beta$-power increases were the most prominent for the frequency band $23.5 \pm$ $3.4 \mathrm{~Hz}$. The individually selected electrodes are indicated overlying the grand-average group topography in Figure $4 A$. To test whether the postmovement $\beta$-rebound was modulated by the amplitudes of the experienced movement-execution errors, we used a mixedeffects linear regression model (see Materials and Methods), which revealed that $\beta$-power was significantly modulated by the size of the kinematic errors during the epoch -184 to $572 \mathrm{~ms}$ around move-
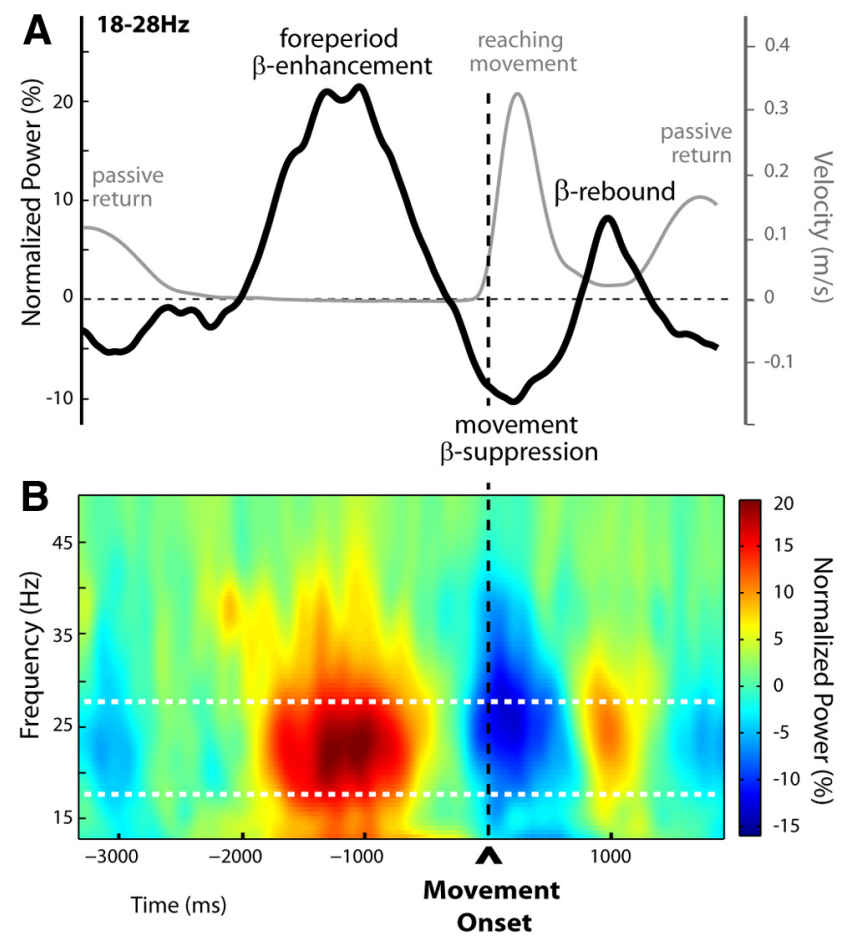

Figure 3. Time-frequency representation of unperturbed trials (Experiment 1). $A$, Group averages of the tangential hand-velocity profiles (gray curve) and $\beta$-power time courses (black curve) were computed for unperturbed trials. Both the EEG and kinematic profiles were obtained by aligning to movement onset. The group $\beta$-power profile is the average over $18-28$ $\mathrm{Hz}$. Two clear peaks in $\beta$-power could be distinguished, separated by a period of oscillatory power decrease. The first $\beta$-power enhancement was observed during the fixed foreperiod between READY and GO (signal occurrences not shown, at approximately -2000 and -500 before movement onset, respectively), throughout which participants maintained their hand stationary at its starting location. The second peak in $\beta$-power reflected the postmovement $\beta$-rebound as usual at the end of movement. The epoch of $\beta$-band suppression (movementrelated $\beta$-ERD) separating these two peaks started before movement onset, reaching a minimum around peak hand tangential velocity. $\boldsymbol{B}$, Group time-frequency map of unperturbed trials aligned to movement onset, obtained by averaging the normalized power over electrodes selected individually for each participant, based on their activity profile during the foreperiod in the unperturbed trials (indicated in Fig. 5A). The foreperiod $\beta$-enhancement and the postmovement $\beta$-rebound are clearly visible, separated by the typical $\beta$-band suppression occurring shortly before and during movement. Note that in $\boldsymbol{A}$ and $\boldsymbol{B}$ the amplitude of the $\beta$-rebound is not optimally represented as signals are aligned to movement onset and not to movement offset.

ment offset, indicated by gray shading in Figure $4 A$; the larger the kinematic error the more attenuated the postmovement $\beta$-power. For illustrative purposes, the group time-frequency maps corresponding to the different sizes of hand-path deviations are shown in Figure $4 B$. These were obtained by averaging over the electrodes that were individually selected, based on their activity observed for the unperturbed trials (see Materials and Methods).

Foreperiod $\beta$-enhancement. During the trial epoch defined relative to movement onset (premovement epoch in trials $n+1$ ), increases in $\beta$-activity in the foreperiod were the most marked for the frequency band $22.6 \pm 2.0 \mathrm{~Hz}$, comparable to those selected for the postmovement epoch $\left(t_{(14)}=0.32, p=0.75\right)$. The individually selected electrodes are indicated over the group topography in Figure 5A.

Mixed-effects linear regressions applied from -3000 to $500 \mathrm{~ms}$ around movement onset (see Materials and Methods) revealed two periods during which $\beta$-power was parametrically modulated by the size of the kinematic errors experienced in the previous trials (the larger the error the more attenuated the $\beta$-power), respectively, 
A Post-movement $\beta$-rebound

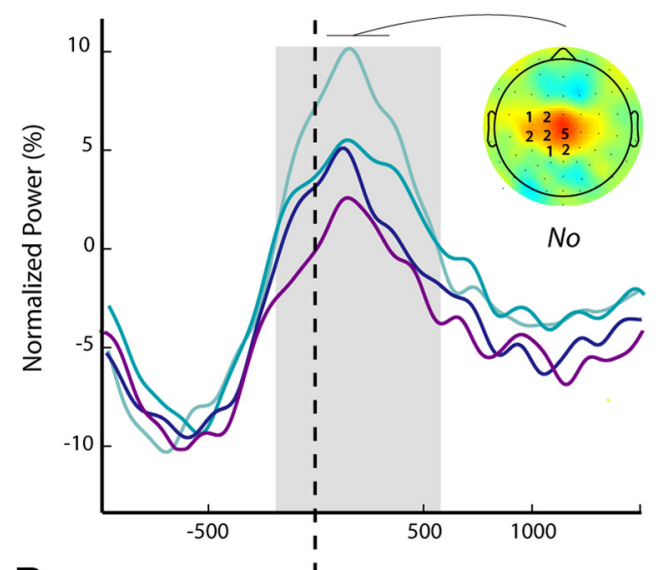

Perturbed trials - No - Small - Medium - Large

B

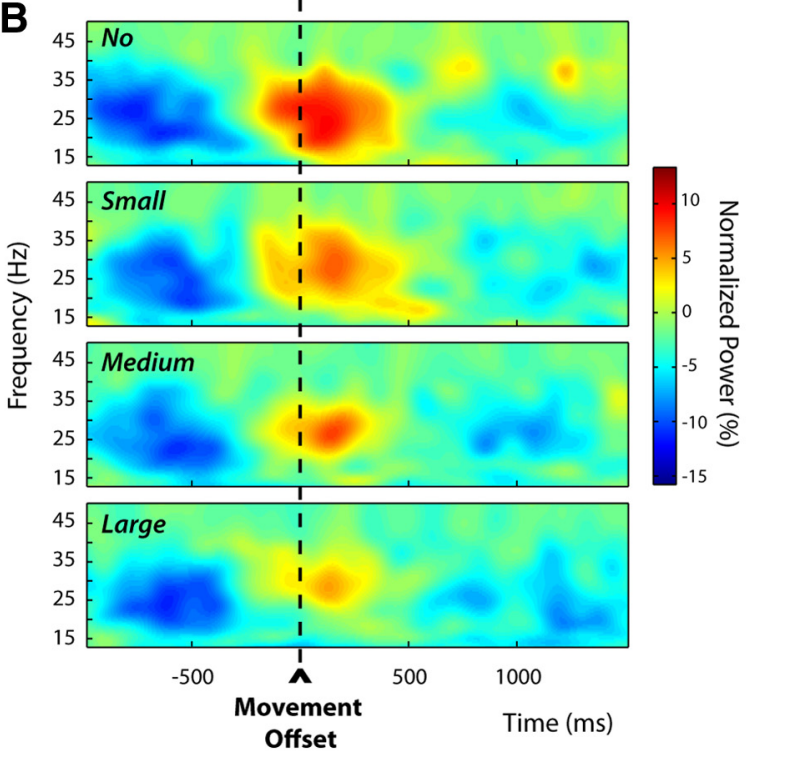

Figure 4. Modulations of the postmovement $\beta$-rebound in perturbed trials $n$ (Experiment 1). $A$, The group average $\beta$-power profiles aligned to movement offset revealed a clear postmovement $\beta$-rebound. These profiles were obtained by averaging the normalized power over the individually selected frequency bands and electrodes (see Materials and Methods); the numbers of participants for which an electrode was selected are indicated superimposed to the group topography computed for the unperturbed trials ( $\mathrm{No}$ ) over a $300 \mathrm{~ms}$ window centered on the peak of the $\beta$-rebound. The period during which the amplitude of the $\beta$-rebound was significantly modulated by the size of the kinematic error is indicated by the gray shading. $\boldsymbol{B}$, Group average time-frequency maps for the unperturbed (No) and the perturbed trials (Small, Medium, and Large) aligned to movement offset, obtained by averaging the normalized powers at the individually selected electrodes as illustrated in $\boldsymbol{A}$.

from -2715 to $-2285 \mathrm{~ms}$ and from -1840 to $-610 \mathrm{~ms}$ (Fig. $5 \mathrm{~A}$, gray shadings).

For illustrative purposes, the group time-frequency maps corresponding to the different sizes of hand-path deviations experienced in the previous trials are presented in Figure 5B. These were obtained by averaging over the electrodes that were individually selected based on their activity observed for the unperturbed trials (see Materials and Methods).

The results from Experiment 1 show that the postmovement $\beta$-rebound was modulated by the amplitudes of movementexecution errors induced by force-field perturbations independently of any motor-correction processes. In addition, they suggest that movement-execution errors in trials $n$ may influence $\beta$-activity during the preparation of the subsequent movements $n+1$. However, they also leave several critical issues unresolved. First, concerning the
A

\section{Foreperiod $\beta$-enhancement}
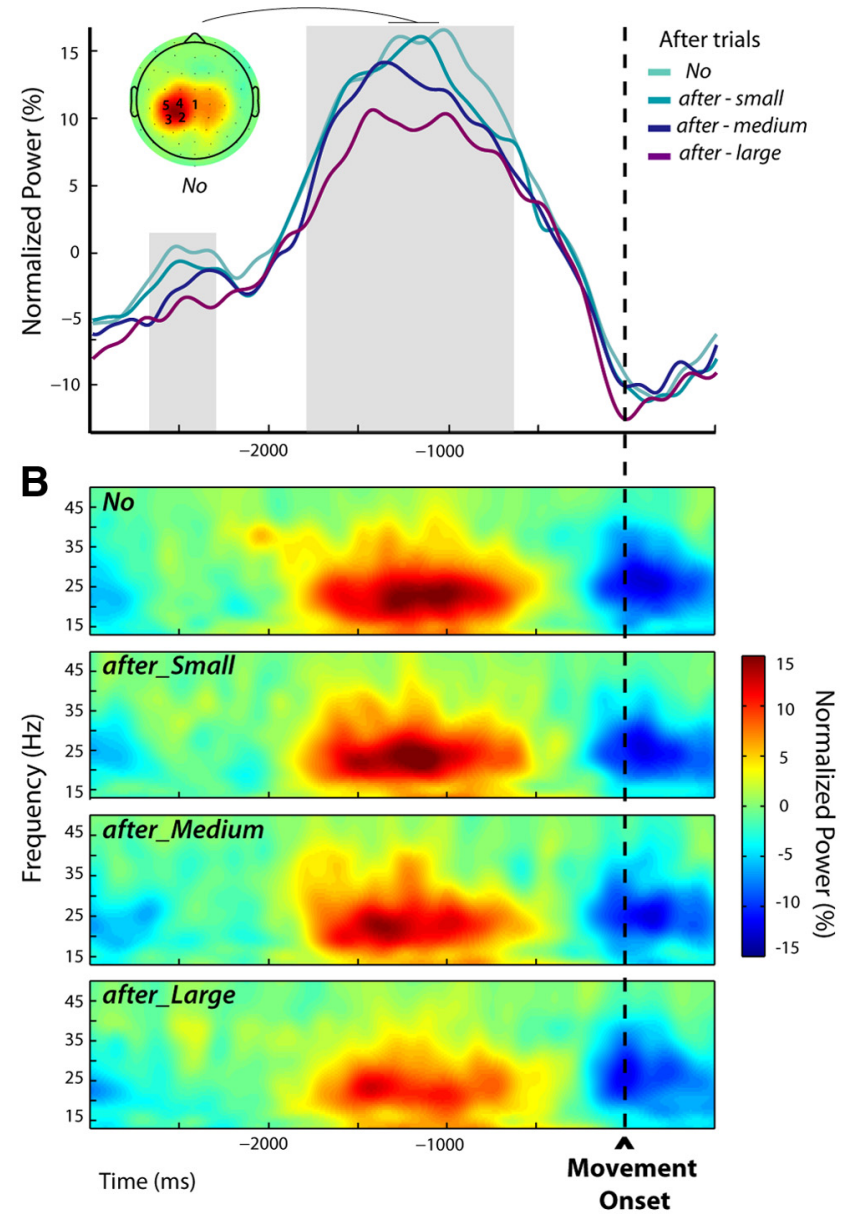

Figure 5. Modulations of the foreperiod $\beta$-enhancement in after trials $n+1$ (Experiment 1). $\boldsymbol{A}$, The group average $\beta$-power profiles exhibited a foreperiod peak followed by a decrease related to the execution of the movement. These profiles were obtained by averaging the normalized powers at the individually selected frequency bands and electrodes (see Materials and Methods); the numbers of participants for which an electrode was selected are indicated superimposed to the group topography computed for the unperturbed trials (№) over a $300 \mathrm{~ms}$ window centered on the peak of the $\beta$-power. The periods during which $\beta$-power was parametrically modulated by the size of the movement-execution error that was experienced in the previous trial are indicated by the gray shadings. $\boldsymbol{B}$, Group average time-frequency maps for the unperturbed ( $\mathrm{No}$ ) and the subsequent trials (after-Small, after-Medium, and after-Large) aligned to movement onset were obtained by averaging the normalized powers at the individually selected electrodes indicated in $\boldsymbol{A}$.

postmovement $\beta$-rebound, based on these results, one cannot determine whether it is modulated in relation to adaptive processes or rather in proportion to the salience of the kinematic errors, independent of any trial-to-trial sensorimotor learning. Second, in the same way, the error-related modulation observed during the foreperiod of the subsequent trials $n+1$ might well correspond to an unspecific alert signal proportional to the surprise aroused by the error experienced in the previous trial $n$, rather than adaptive motor-command adjustments of the upcoming movement. Experiment 2 was designed to remove these ambiguities.

\section{Experiment 2, protocol 1}

Behavioral performance

In this experiment, participants performed a pointing task in which two different types of reach errors were contrasted: movementexecution errors that elicited trial-to-trial motor-command adjust- 


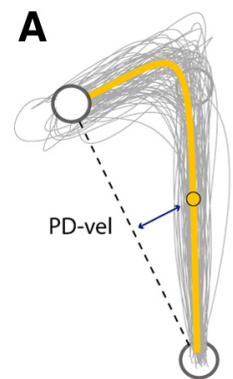

Target Jump

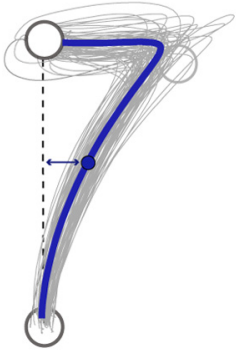

Force field

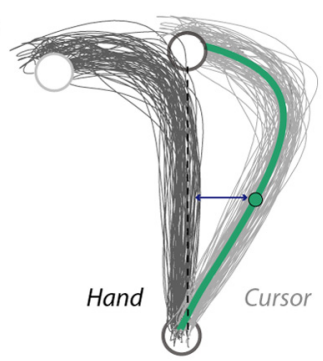

Visual Rotation
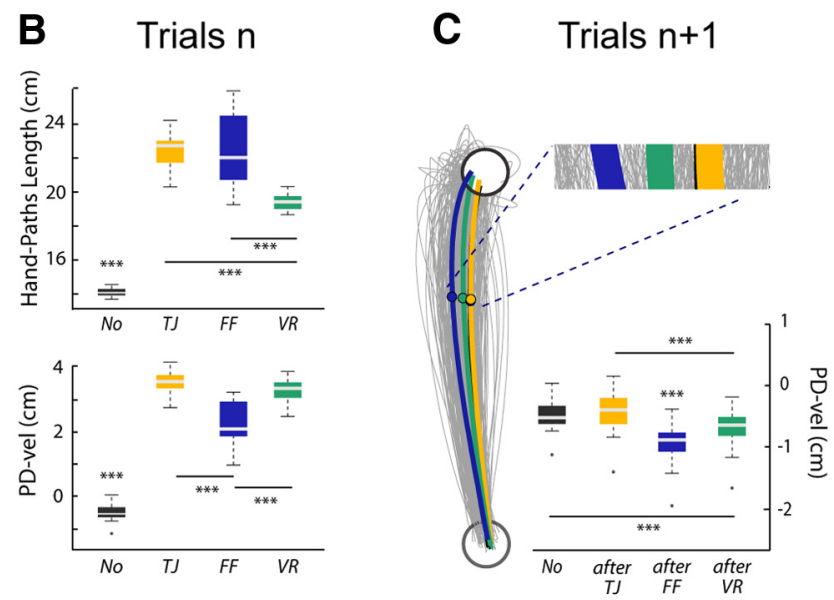

Figure 6. Behavioral data from the pointing task (Experiment 2, Protocol 1). $\boldsymbol{A}$, For a representative participant, individual and average hand paths for the three categories of perturbed trials (Target Jump, Force Field, and Visual Rotation) showing clear on-line movement corrections. The starting position and the target are plotted in black. For the Visual Rotation trials, the actual hand paths are plotted in black and the paths of the finger-tip cursor are shown in gray. $\boldsymbol{B}$, Boxplots summarizing the results for the PD-vel and the HPL (***p $<0.001)$. C, For the same participant, individual and average hand paths for the different subsequent trials (after-TJ, after-FF, and after-VR). Group data for the PD-vel and statistical results are summarized in the boxplot ( $\left.{ }^{* * *} p<0.001\right)$.

ments, and goal errors that did not trigger sensorimotor adaptation (Diedrichsen et al., 2005). Movement-execution errors were produced by mechanical (force-field) or visual (rotation) perturbations, whereas goal errors were provoked by unexpected displacements of the target upon movement initiation. In contrast to Experiment 1, participants were instructed to end their movements within the target, so on-line corrections were clearly observed in all the perturbed trials, as illustrated by the individual hand paths plotted in Figure $6 \mathrm{~A}$. As for Experiment 1, reaction times were similar for the perturbed $n$, after $n+1$ and unperturbed No trials (No $=497 \pm 76 \mathrm{~ms}$, perturbed: $492 \pm 77 \mathrm{~ms}$, and after trials: $478 \pm 75 \mathrm{~ms}, F_{(2,26)}: 1.44, p=$ $0.26)$, and differences were only observed for movement durations $\left(F_{(2,26)}=449.7, p<0.001\right)$. Movements lasted longer in perturbed trials $(700 \pm 14 \mathrm{~ms})$ relative to both $\mathrm{No}\left(401 \pm 4 \mathrm{~ms}, t_{(13)}=21.9, p<\right.$ $0.001)$ and after trials $\left(423 \pm 4 \mathrm{~ms}, t_{(13)}=21.1, p<0.001\right)$. Movements also lasted longer in after trials than in No trials $\left(t_{(13)}=6.1 p<\right.$ $0.001)$.

To quantify reach error in trials $n$, in addition to the PD-vel, we used a complementary measure: the HPL (see Materials and Methods). A repeated-measures MANOVA revealed a significant effect of trial category, TJ, FF, and VR, on the two kinematic-error measures, PD-vel and HPL $\left(F_{(3,39)}=122.2, p<0.001\right)$. Follow-up univariate analyses confirmed that, while these two indices were highly correlated $(\rho=0.88, p<0.001)$, they were not redundant, and allowed to capture distinct features of reach errors. Both univariate repeated-measures ANOVAs were significant $\left(\mathrm{PD}\right.$-vel: $F_{(3,39)}=575.99 ; \mathrm{HPL}: F_{(3,39)}=140.7 ; p<0.001$, in both cases), but the associated post hoc pairwise comparisons revealed distinct patterns (Fig. 6B). PD-vels were significantly larger for all the perturbed relative to the unperturbed No trials (FF vs No: $t_{(13)}=-18$, VR vs No: $t_{(13)}=-52.5$, TJ vs No: $t_{(13)}=$ 44.9; $p<0.001$, in all cases). In addition, larger PD-vels were observed in VR and TJ trials than in FF trials (VR vs FF: $t_{(13)}=8.7$ and TJ vs FF: $t_{(13)}=9.9, p<0.001$ in both cases). Quantifying reach errors by the HPL offered a different picture. While all the catch trials also clearly differed from the unperturbed trials (FF vs No: $t_{(13)}=13.3$, VR vs No: $t_{(13)}=26.5$, TJ vs No: $t_{(13)}=26.6$; $p<$ 0.001 , in all cases) larger HPLs were observed in both TJ and FF trials relative to VR trials (TJ vs VR: $t_{(13)}=9.9, p<0.001$ and FF vs VR: $t_{(13)}=5.8 ; p<0.001$ in both cases). One may notice, however, that both univariate analyses pointed to TJ trials as exhibiting the largest reach errors.

To investigate the trial-to-trial adaptive responses, visible in trials $n+1$, associated with the different types of reach errors, we contrasted the kinematics of the No, after-TJ, after-FF, and after-VR trials. Illustrative individual hand paths and group data are presented in Figure 6C. A repeated MANOVA run on both kinematic error measures (PD-vel and HPL) revealed a significant effect of the category of trials $n+1{\left(F_{(3,39)}\right.}=21.9, p<$ $0.001)$. The following univariate repeated-measures ANOVAs revealed significant results only for the PD-vel measure $\left(F_{(3,39)}=\right.$ 66.5, $p<0.001)$. Further, hand-path deviations were larger for after-FF and after-VR trials than for No and after-TJ trials (after-FF vs No: $t_{(13)}=-12.4$; after-VR vs No: $t_{(13)}=-6.1$; after-FF vs after-TJ: $t_{(13)}=-10.5$; after-VR vs after-TJ: $t_{(13)}=$ $-6.4, p<0.001$ in all cases). Deviations were larger for after-FF than for after-VR trials $\left(t_{(13)}=-6.1, p<0.001\right)$. No significant difference was observed between No and after-TJ trials $\left(t_{(13)}=\right.$ $3.4, p=0.06)$.

\section{EEG time-frequency data}

As can be seen in Figures $7 A$ and $8 A$, the $\beta$-power profiles observed during the two trial epochs of interest (postmovement and premovement epochs) exhibited time courses and scalp locations similar to those observed in the previous experiment. Again, $\beta$-peaks occurred within similar frequency bands for both epochs (mean of frequency-band centers $=22.3 \pm 3.2$ and $23.1 \pm 2.2$ $\mathrm{Hz}$, for the postmovement and premovement epochs, respectively; $t_{(13)}=0.34, p=0.74$ ).

Postmovement $\beta$-rebound. As in Experiment 1, within the postmovement epoch, a long period extending from -10 to 670 ms around movement offset could be identified during which the category of trials had a significant effect (Fig. $7 A$, gray shading). In all perturbed trials $n, \mathrm{TJ}, \mathrm{FF}$, and $\mathrm{VR}, \beta$-power was reduced relative to the unperturbed No trials. Post hoc pairwise comparisons revealed that the different reach errors attenuated $\beta$-power over slightly different time windows (TJ vs No: -10 to $670 \mathrm{~ms}$, FF vs No: -10 to $350 \mathrm{~ms}$, VR vs No: -10 to $80 \mathrm{~ms}$ and 100 to $270 \mathrm{~ms}$; Fig. $7 A$, colored bars). Figure $7 B$ presents the group timefrequency maps for the four conditions time locked to the movement offset, obtained by averaging over the individually selected electrodes (see Materials and Methods).

Foreperiod $\beta$-enhancement. The repeated-measures ANOVAs performed over the premovement epoch allowed us to identify two short and one long period during which $\beta$-activity differed significantly between trial categories; that is, depending on the size of the movement error experienced in the previous trial. These periods were in the foreperiod, and extended respectively 
A
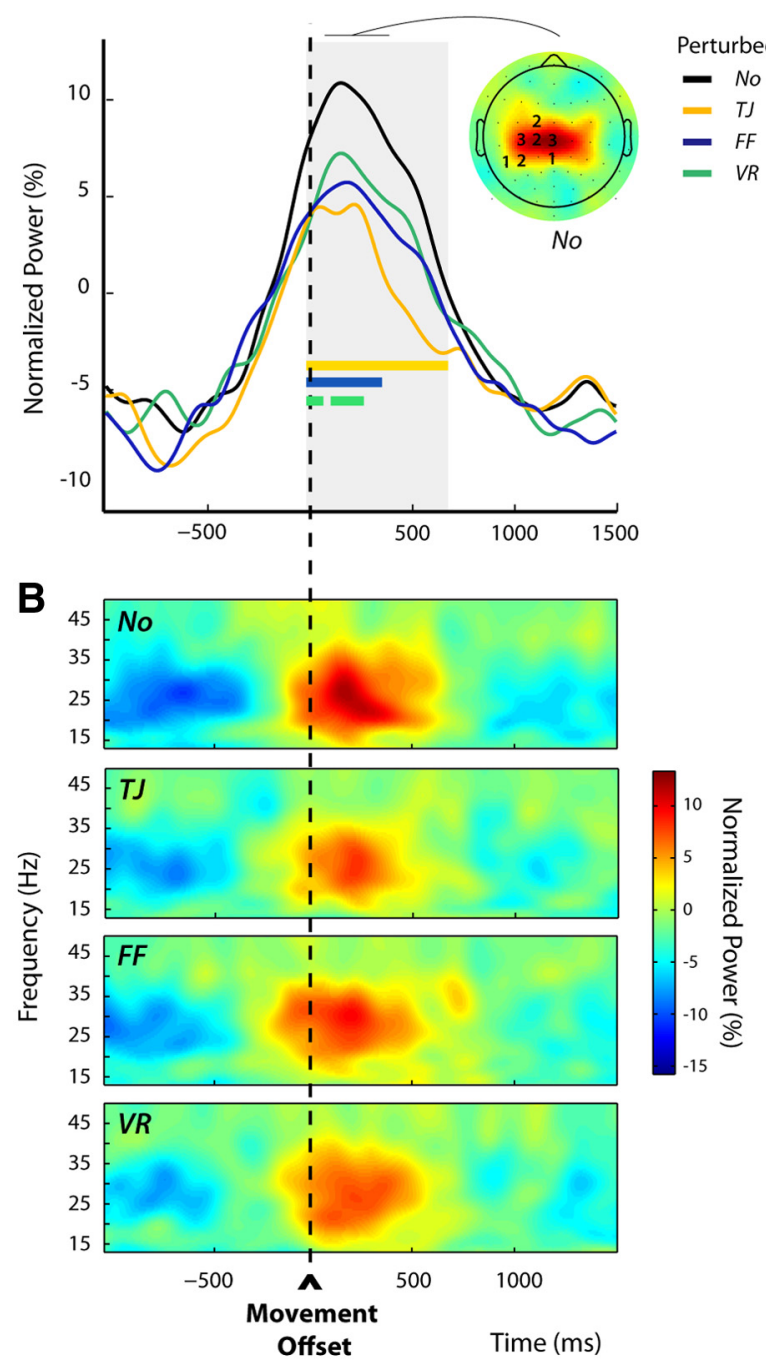

Figure 7. Modulations of the postmovement $\beta$-rebound in perturbed trials $n$ (Experiment2, Protocol 1). $\boldsymbol{A}$, Group average $\beta$-power profiles aligned to movement offset for the different categories of trials: No, TJ, FF, and VR. These were obtained by averaging over the frequency bands and electrodes individually selected (see Materials and Methods); the numbers of participants for which an electrode was selected are indicated superimposed to the group topography computed for the unperturbed trials (No) over a 300 ms window centered on the peak of the $\beta$-rebound. The period during which $\beta$-power differed significantly between trial categories is indicated by the gray shading. For each category of perturbed trials (TJ, FF, and VR), the epoch during which $\beta$-power differed significantly from that observed for the unperturbed trials ( $\mathrm{N}_{0}$ ) is indicated by a colored bar. An attenuation of the $\beta$-rebound was observed after all types of perturbations. $\boldsymbol{B}$, Group average time-frequency maps for the unperturbed (No) and the perturbed (TJ, FF, and VR) trials aligned to movement offset, obtained by averaging the normalized power over the individually selected electrodes as illustrated in $\boldsymbol{A}$.

from -2045 to $-1955 \mathrm{~ms}$, from -1775 to $-1665 \mathrm{~ms}$, and from -1225 to $-875 \mathrm{~ms}$ relative to movement onset (Fig. $8 \mathrm{~A}$ ). In post hoc pairwise tests, each category of after trials (after-TJ, afterFF, and after-VR) was compared with the unperturbed No condition. In both after-FF and after-VR trials $\beta$-power was significantly reduced relative to No trials, but during different epochs (after-FF vs No: -1165 to $-875 \mathrm{~ms}$, after-VR vs No: -1775 to $-1665 \mathrm{~ms}$, and -1225 to $-975 \mathrm{~ms}$; Fig. 8 A, colored bars). In contrast, the $\beta$-power profiles observed for the after-TJ trials at no time significantly differed from those for the No trials; that is, $\beta$-power was attenuated only in trials that directly followed movement-execution errors, not following goal

\section{A \\ Foreperiod $\beta$-enhancement}

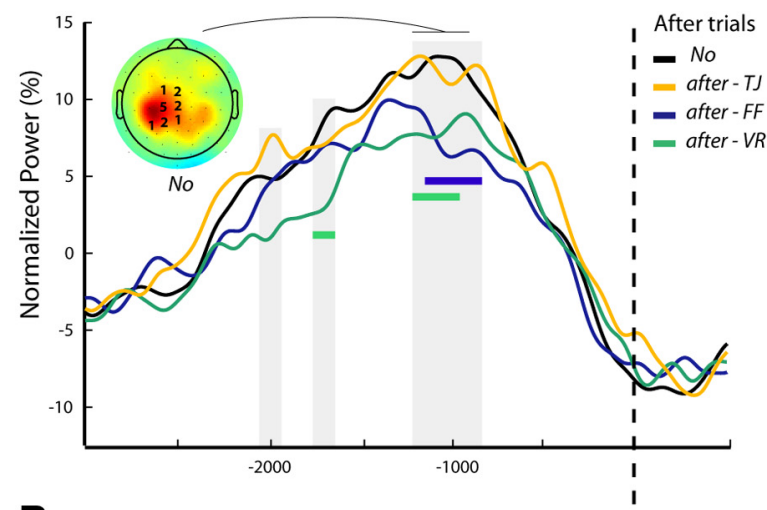

B
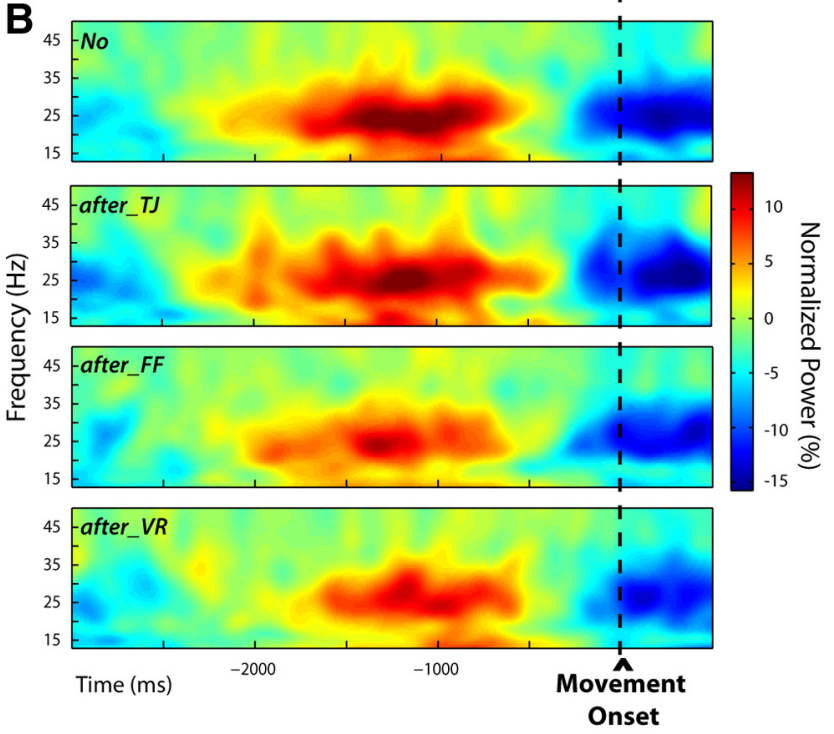

Figure 8. Modulations of the foreperiod $\beta$-enhancement in after trials $n+1$ (Experiment 2, Protocol 1). A, Group average $\beta$-power profiles aligned to movement onset for the different categories of trials: No, after-TJ, after-FF, and after-VR. These were obtained by averaging over individually selected frequency bands and electrodes (see Materials and Methods); the numbers of participants for which an electrode was selected are indicated superimposed to the group topography computed for the unperturbed trials (No) over a 300 ms window centered on the peak of the $\beta$-power. The periods during which $\beta$-power differed significantly between trial categories are indicated by gray shadings. An attenuation of the foreperiod $\beta$-power was observed when movement-execution errors were experienced in the previous trial (after-FF and after-VR) but not when a goal error (after-TJ) was induced. The periods during which $\beta$-power was significantly reduced for the after-FF and after-VR trials are indicated by colored bars. $\boldsymbol{B}$, Group average time-frequency maps for the unperturbed (№) and the subsequent trials (after-TJ, after-FF, and after-VR) aligned to movement onset were obtained by averaging the normalized powers at the individually selected electrodes indicated in $\boldsymbol{A}$.

errors. Figure $8 B$ shows the group time-frequency maps corresponding to the different conditions obtained by averaging over the individually selected electrodes (see Materials and Methods).

\section{Experiment 2, protocol 2}

To limit the temporal overlap between functionally distinct $\beta$-components, in the second protocol we increased the duration of the delay between the passive return to the starting position (at the end of trial $n$ ) and the warning READY cue (at the start of trial $n+1$ ) from $500 \mathrm{~ms}$ (Protocol 1) to $2000 \mathrm{~ms}$. Our goal was to separate any effects on the $\beta$-power resulting from the end-oftrial passive return from those related to the planning of the subsequent movement. Participants performed the same task as in the first protocol; however, with reach errors induced only by mechanical perturbations (force field). 


\section{Behavioral performance}

As in the previous experiments, clear reach errors were observed in the perturbed catch trial $n$ relative to unperturbed No trials (FF vs No, $\left.F_{(2,8)}=66.7, t^{2}=150.1, p<0.001\right)$. Both kinematic measures revealed significant deviations induced by mechanical perturbations (PD-vel: No: $-0.5 \pm 0.1 \mathrm{~cm}, \mathrm{FF}: 2 \pm 0.3 \mathrm{~cm}$, $t_{(9)}=-10.7$, HPL: No: $14 \pm 0.1 \mathrm{~cm}$, FF: $20.8 \pm 1.3 \mathrm{~cm}$, $t_{(9)}=-4.85, p<0.001$ in both cases). Also congruent with previous observations, the after-FF trials exhibited slight but significant deviations relative to the No trials (after-FF vs No, $F_{(2,8)}=$ 26.3, $\left.t^{2}=59.2, p<0.001\right)$. These significant hand-path deviations were only revealed by the $\mathrm{PD}$-vel measures (PD-vel: No: $-0.5 \pm 0.1 \mathrm{~cm}$, after-FF $\left.=-0.8 \pm 0.2 \mathrm{~cm}, t_{(9)}=-10.7, p<0.001\right)$.

\section{EEG time-frequency data}

Postmovement $\beta$-rebound. In the unperturbed No trials a clear $\beta$-rebound was visible around movement offset with a frontocentral topography (Fig. 9A). The $\beta$-power increase was the most prominent for the frequency band $21.6 \pm 3.5 \mathrm{~Hz}$. Consistent with the previous experiments, statistical analyses confirmed a significant attenuation of the $\beta$-power after mechanical perturbations in two time windows extending from -70 to $20 \mathrm{~ms}$ and 100 to $510 \mathrm{~ms}$ around movement offset (Fig. 9A, gray shading).

Foreperiod $\beta$-enhancement. Due to the longer delay (2000 ms) between the end of the passive return in trial $n$ and the onset of the active movement in trial $n+1$, the $\beta$-power patterns differed from those observed in the first protocol (Fig. 9B). While in Protocol 1 a single peak was observed during the foreperiod (Fig. $8 A$ ), here two peaks were distinguishable for the unperturbed No trials: a first large one clearly lateralized, peaking before the READY signal marking the start of the foreperiod and a second smaller one maximal above the parietocentral electrodes during the foreperiod. This second peak was absent for the after-FF trials (Fig. $9 B)$. The frequency band with the most marked effect was $23.1 \pm$ $1.2 \mathrm{~Hz}$ (not significantly different from the frequency bands selected for the rebound $\left(t_{(9)}=0.45, p=0.67\right)$.

Statistical tests revealed that the $\beta$-power significantly differed between the two conditions only over a time window extending from -1330 to $-750 \mathrm{~ms}$, coinciding with the timing of the second peak in the unperturbed No trials. There was no difference between conditions during the preceding larger peak. These results confirm our supposition that the foreperiod modulations observed in the previous experiments truly affect a $\beta$-component related to movement preparation processes, independent of a preceding nonmodulated component possibly related to the passive return movement. For illustrative purposes, the group time-frequency maps time locked to the movement onset for the after-FF and No trials are presented in Figure 9B. These were computed using the $\beta$-activity recorded at the electrodes individually selected according to their sensitivity to trial category (see Materials and Methods).

\section{Discussion}

In the present study, we report two main findings. First, the attenuation of the cortical $\beta$-rebound after movement errors may relate to error salience independently of sensorimotor adaptation mechanisms and in the absence of on-line corrections. Second, and in contrast, modulations in $\beta$-activity during movement preparation in trials succeeding a perturbed trial may relate to neural processes involved in motor command adjustment.

In the first experiment, consistent with Tan et al. (2014a,b), we found that the postmovement $\beta$-rebound was parametrically attenuated by movement-execution errors produced by mechanical per- turbations. By using a task in which participants experienced movement errors without having the opportunity to correct for them, we furthermore demonstrate that this modulation does not depend on corrective movements proportionate to the induced errors. Importantly, we also examined $\beta$-power during movement preparation and found that it was attenuated when a movementexecution error was experienced in the preceding trial. It is well known that, in addition to reach errors in the perturbed catch trials, mechanical perturbations induce adaptive responses that are visible in the subsequent trials as slight hand-path deviations in the opposite direction (Thoroughman and Shadmehr, 2000). The observed changes in foreperiod $\beta$-activity might reflect such motorcommand adjustment processes.

While the design of Experiment 1 was intended to dissociate error processing from on-line motor correction, it did not permit determining whether the modulations of the foreperiod and postmovement $\beta$-activities reflect trial-to-trial sensorimotor adaptation or "merely" relate to unspecific responses proportional to the salience of the kinematic errors. This is also a limit of the paradigm used by Tan et al. (2014a,b). The goal of the second experiment was to remove an important ambiguity, dissociating the reach error experienced in the perturbed trial from the motor-command adjustment observed in the subsequent trial. We contrasted two types of reach errors to accomplish this dissociation: movement-execution errors that triggered adaptive mechanisms and goal errors that produced comparable hand-path deviations but elicited no sensorimotor adaptation (Diedrichsen et al., 2005). The foreperiod and postmovement $\beta$-activities exhibited clearly distinct patterns: the postmovement $\beta$-rebound was attenuated following both types of reach errors, whereas the foreperiod $\beta$-activity was impacted only by reach errors driving sensorimotor adaptation.

\section{The postmovement $\boldsymbol{\beta}$-rebound and error salience}

In a visuomotor adaptation task, Tan et al. (2014a,b) demonstrated the modulation of the $\beta$-rebound by movementexecution errors. By manipulating the context of experienced kinematic errors (constant vs random perturbations), they showed that the effect of an error of a given size varies depending on how noticeable it is given the context; that is, the salience of the error, rather than its sensory-encoded amplitude, impacts the $\beta$-rebound. However, in their study (as in our first experiment) error salience and sensorimotor adjustment covary. As a consequence one cannot determine which component is critical.

In our second experiment, we observed that the $\beta$-rebound was also reduced by goal errors, which do not trigger sensorimotor adaptation (Diedrichsen et al., 2005). Tan et al. $(2014 a, b)$ proposed that the $\beta$-rebound reflects neural processes that evaluate motor errors consistent with Bayesian inference involved in sensorimotor adaptation. The results of our second experiment demand an interpretative framework general enough to also incorporate the modulation of the $\beta$-rebound in response to unexpected events that do not result in sensorimotor remapping and subsequent changes in the motor command.

Furthermore, error-related modulations of the $\beta$-rebound were also reported by Koelewijn et al. (2008) in a study using a response-selection task. Interestingly, these authors found an enhanced rather than attenuated postmovement $\beta$-rebound following erroneous responses. We can only speculate that differences in the source (internal or external) of the error signal might explain this apparently conflicting result. In the cognitive task used by Koelewijn et al. (2008), the error signal arises from the comparison between the actual movement un- 
A

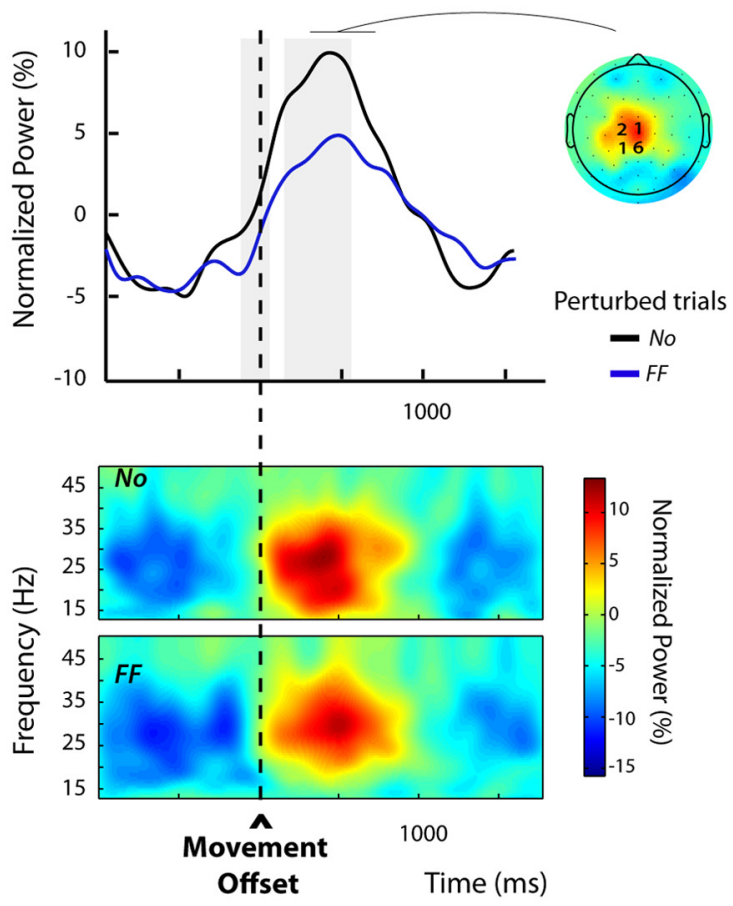

B

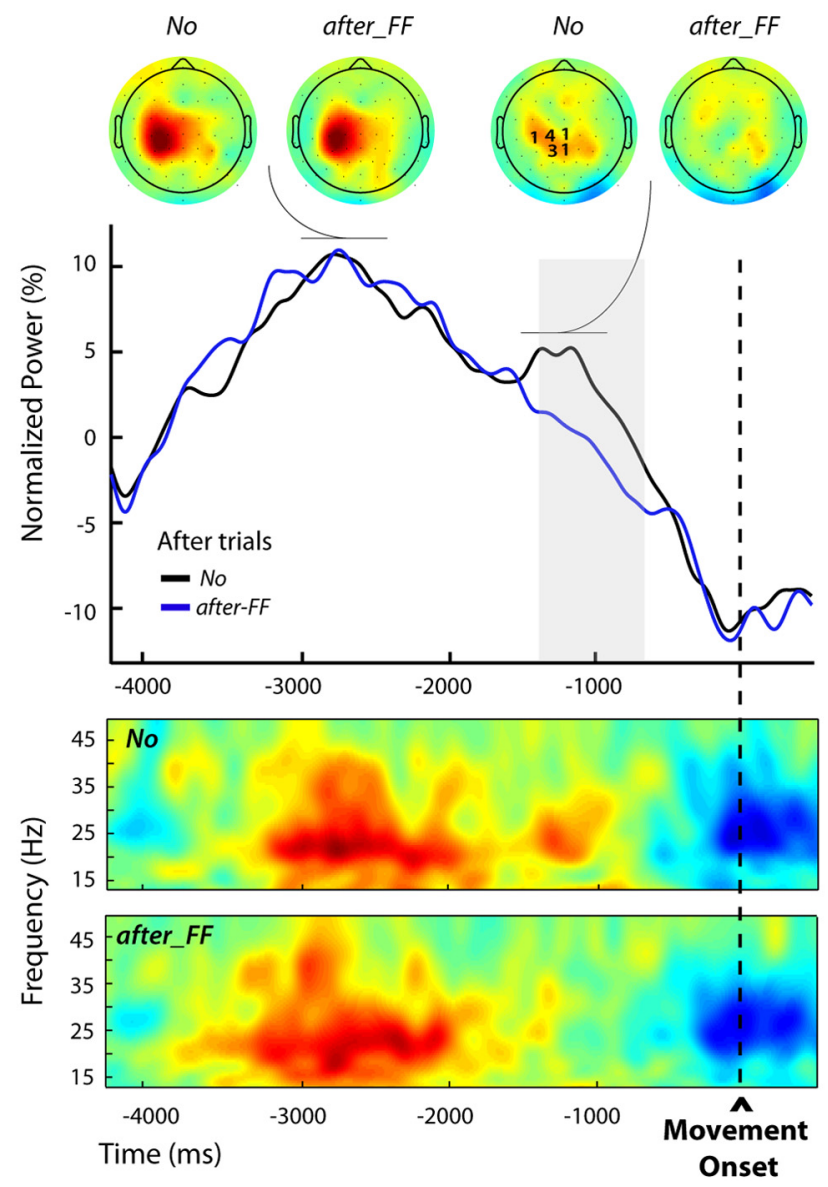

Figure 9. Modulations of the postmovement and foreperiod $\beta$-enhancements (Experiment 2, Protocol 2). A, Top, Group average $\beta$-power profiles aligned to movement offset for the folding and the internally represented correct response. The authors proposed that "the beta rebound we observe following errors reflects active inhibition of ongoing motor processes: the stronger rebound accompanying the generation of an error reflects response inhibition that typically follows the detection of an erroneous action" (Ridderinkhof et al., 2004). In contrast, in Tan et al. (2014a,b) and in our experiments, participants encode and process external, unexpected sensory feedbacks.

\section{Foreperiod $\boldsymbol{\beta}$-activity and motor-command updating}

The gradual decrease in $\beta$-power immediately preceding movement initiation has been extensively described (Nagamine et al., 1996; Pfurtscheller and Lopes da Silva, 1999; Taniguchi et al., 2000; Paradiso et al., 2004). In contrast, only a handful of studies have analyzed the modulation of $\beta$-activity further back in time during the foreperiod (for review, see Kilavik et al., 2013). Using precueing paradigms several studies have reported intermittent $\beta$-band peaks during the foreperiod between the warning and the imperative cues (Alegre et al., 2004, 2006; Molnár et al., 2008; van Wijk et al., 2009; Fischer et al., 2010; Zaepffel et al., 2013). First, Alegre et al. $(2004,2006)$ reported multiple, largely independent $\beta$-power peaks in this epoch, of frontal and central sources. Recently, in a precued reach-to-grasp task, Zaepffel et al. (2013) found that $\beta$-band power was reduced above contralateral sensorimotor cortex following cues that contained information about grip type. This suggests that $\beta$-activity in this epoch is sensitive to movement preparation processes (Kilavik et al., 2013). However, in the present study the warning READY cue in itself did not carry information relevant for the generation of a new motor plan, it only served as a temporal warning cue that the participants could use to start (re-)planning the movement, since the foreperiod duration was fixed.

In our second experiment (Protocol 1 ), foreperiod $\beta$-power was attenuated only when a movement-execution error driving sensorimotor adaptation was experienced in the preceding trial, suggesting that it is related to remapping mechanisms. However, one could have suspected that a $\beta$-component related to the passive return at the end of trial might have contributed to the observed effect. The increased delay between the end of the passive return and the READY signal in the second protocol of Experiment 2 allowed ruling out this possibility. Here, two $\beta$-components could be clearly distinguished: a first component maximum $\sim 1$ s after the

\footnotetext{
$\leftarrow$

unperturbed ( $\mathrm{No}$ ) and perturbed trials (FF), obtained by averaging over the individually selected frequency bands and electrodes (see Materials and Methods); the numbers of participants for which an electrode was selected are indicated superimposed to the group topography computed for the unperturbed trials (No) over a $300 \mathrm{~ms}$ window centered on the peak of the $\beta$-rebound. The period during which $\beta$-power differed significantly between the two categories of trials is indicated by the gray shading. Bottom, Group average time-frequency maps for both categories of trials (No and FF) aligned to movement offset, obtained by averaging the normalized power at the individually selected electrodes. $\boldsymbol{B}$, Top, Group average $\beta$-power profiles aligned to movement onset for the unperturbed (No) and after-FF trials. These profiles were obtained by averaging over the individually selected frequency bands and electrodes (see Materials and Methods); the numbers of participants for which an electrode was selected are indicated superimposed to the group topography computed for the unperturbed trials (No) over a $300 \mathrm{~ms}$ window centered on the second smaller peak in $\beta$-power. The period during which $\beta$-power differed significantly between the two trial categories is indicated by the gray shading. Group topographies are shown for both categories of trials (No) and after trials (FF), computed over two different 300 ms windows centered, respectively, on the first and second $\beta$-power peak visible in the unperturbed (No) trials. Bottom, Group average time-frequency maps for the $\mathrm{No}$ and the after-FF trials, aligned to movement onset, obtained by averaging the normalized powers at the individually selected electrodes.
} 
passive return probably reflecting, at least in part, a $\beta$-rebound (Cassim et al., 2001; Alegre et al., 2002; Müller et al., 2003), followed by a smaller component visible in the reference No trials as a deflection arising around $1200 \mathrm{~ms}$ before movement onset. Clearly only this latter $\beta$-component was modulated with motor-command updating subsequent to movement-execution errors experienced in the previous trials. Its attenuation in relation to sensorimotor remapping fits well with theories proposing that the level of $\beta$-activity provides a running index of the extent to which internal or external cues predict the need for action or action updating (Jenkinson and Brown, 2011).

\section{Processing of salient sensory events and motor adaptation are both reflected in $\boldsymbol{\beta}$-power attenuation}

Converging evidence suggests that $\beta$-power relates inversely to the efficacy with which sensory information is processed (Linkenkaer-Hansen et al., 2004; Zhang et al., 2008; van Ede et al., 2010, 2011) and to cortical excitability (Chen et al., 1998 Tamura et al., 2005). However, recent studies also show that sensorimotor $\beta$-power peaks at moments when salient sensory events are expected (Saleh et al., 2010; Takahashi et al., 2011; Fujioka et al., 2012; Zaepffel et al., 2013). The mechanisms that underpin these seemingly contradictory observations are largely unknown.

Oscillatory power is related to the extent of rhythmic synchronization of spiking activity (Baker et al., 1997, 1999; Denker et al., 2011; Engelhard et al., 2013). Under some circumstances a rhythmically synchronous processing mode might be optimal, providing an efficient movement encoding with as little cortical activity as possible, while limiting the cortical processing capacity (Zohary et al., 1994; Baker et al., 1997, 1999). This mode of processing might be sufficient when repeating the same movement across many trials. In other cases a more asynchronous processing mode might be needed to increase the processing capacity or signal-to-noise ratio (Zohary et al., 1994; Baker et al., 1997, 1999), allowing efficient processing of unexpected salient changes in the sensory feedback as well as adaptive modifications to the motor program. These different processing modes might be implemented in several different networks, in relation to the specific encoding.

Our findings demonstrate distinct modulations in sensorimotor postmovement and foreperiod $\beta$-enhancements related to error salience and sensorimotor adaptation. They point to functional differences in the underlying neuronal activities, probably supported by distinct networks, which might be reflected in different topographies and frequency bands. While we found that the frequency bands associated with the two $\beta$-components were largely overlapping, the data of our last experiment (Experiment 2, Protocol 2) might suggest slightly different localizations, with a more medial peak for the postmovement $\beta$-rebound than for the foreperiod $\beta$-component, slightly more lateralized.

Several previous studies have examined $\beta$-oscillatory correlates of feedback processing in the context of rewardlearning tasks, and have reported frontal or mid-frontal topographies (for review, see Luft, 2014). In particular, it has been reported that unexpected rewards were associated with $\beta$-power increases in these regions (Marco-Pallares et al., 2008, 2015; HajiHosseini et al., 2012). On the other hand, the few studies in which the feedback represented the accuracy of the response rather than a reward have found $\beta$-desynchronization over the contralateral sensorimotor areas following error feedback (van de Vijver et al., 2011; Luft et al., 2013, 2014). Further research will be needed to determine how our findings may (or may not) relate to these two distinct forms of $\beta$-oscillatory modulations.

\section{References}

Aguera PE, Jerbi K, Caclin A, Bertrand O (2011) Elan: a software package for analysis and visualization of MEG, EEG, and LFP signals. Comput Intell Neurosci 2011:158970. CrossRef Medline

Alegre M, Labarga A, Gurtubay IG, Iriarte J, Malanda A, Artieda J (2002) Beta electroencephalograph changes during passive movements: sensory afferences contribute to beta event-related desynchronization in humans. Neurosci Lett 331:29-32. CrossRef Medline

Alegre M, de Gurtubay IG, Labarga A, Iriarte J, Malanda A, Artieda J (2004) Alpha and beta oscillatory activity during a sequence of two movements. Clin Neurophysiol 115:124-130. CrossRef Medline

Alegre M, Imirizaldu L, Valencia M, Iriarte J, Arcocha J, Artieda J (2006) Alpha and beta changes in cortical oscillatory activity in a go/no go randomly-delayed-response choice reaction time paradigm. Clin Neurophysiol 117:16-25. CrossRef Medline

Baker SN, Olivier E, Lemon RN (1997) Coherent oscillations in monkey motor cortex and hand muscle EMG show task-dependent modulation. J Physiol 501:225-241. CrossRef Medline

Baker SN, Kilner JM, Pinches EM, Lemon RN (1999) The role of synchrony and oscillation in the motor output. Exp Brain Res 128:109-117. CrossRef Medline

Burge J, Ernst MO, Banks MS (2008) The statistical determinants of adaptation rate in human reaching. J Vis 8(4):20.1-19. CrossRef Medline

Buschman TJ, Miller EK (2007) Top-down versus bottom-up control of attention in the prefrontal and posterior parietal cortices. Science 315: 1860-1862. CrossRef Medline

Cassim F, Monaca C, Szurhaj W, Bourriez JL, Defebvre L, Derambure P, Guieu JD (2001) Does post-movement beta synchronization reflect an idling motor cortex? Neuroreport 12:3859-3863. CrossRef Medline

Chen R, Yaseen Z, Cohen LG, Hallett M (1998) Time course of corticospinal excitability in reaction time and self-paced movements. Ann Neurol 44: 317-325. CrossRef Medline

Classen J, Gerloff C, Honda M, Hallett M (1998) Integrative visuomotor behavior is associated with interregionally coherent oscillations in the human brain. J Neurophysiol 79:1567-1573. Medline

Delorme A, Sejnowski T, Makeig S (2007) Enhanced detection of artifacts in EEG data using higher-order statistics and independent component analysis. Neuroimage 34:1443-1449. CrossRef Medline

Denker M, Roux S, Lindén H, Diesmann M, Riehle A, Grün S (2011) The local field potential reflects surplus spike synchrony. Cereb Cortex 21: 2681-2695. CrossRef Medline

Diedrichsen J, Hashambhoy Y, Rane T, Shadmehr R (2005) Neural correlates of reach errors. J Neurosci 25:9919-9931. CrossRef Medline

Engel AK, Fries P (2010) Beta-band oscillations-signalling the status quo? Curr Opin Neurobiol 20:156-165. CrossRef Medline

Engelhard B, Ozeri N, Israel Z, Bergman H, Vaadia E (2013) Inducing gamma oscillations and precise spike synchrony by operant conditioning via brain-machine interface. Neuron 77:361-375. CrossRef Medline

Fine MS, Thoroughman KA (2007) Trial-by-trial transformation of error into sensorimotor adaptation changes with environmental dynamics. J Neurophysiol 98:1392-1404. CrossRef Medline

Fischer T, Langner R, Diers K, Brocke B, Birbaumer N (2010) Temporospatial dynamics of event-related EEG beta activity during the initial contingent negative variation. PLoS One 5:e12514. CrossRef Medline

Fujioka T, Trainor LJ, Large EW, Ross B (2012) Internalized timing of isochronous sounds is represented in neuromagnetic Beta oscillations. J Neurosci 32:1791-1802. CrossRef Medline

Gilbertson T, Lalo E, Doyle L, Di Lazzaro V, Cioni B, Brown P (2005) Existing motor state is favored at the expense of new movement during $13-35 \mathrm{~Hz}$ oscillatory synchrony in the human corticospinal system. J Neurosci 25:7771-7779. CrossRef Medline

Goodale MA, Pelisson D, Prablanc C (1986) Large adjustments in visually guided reaching do not depend on vision of the hand and perception of target displacement. Nature 320:748-750. CrossRef Medline

HajiHosseini A, Rodríguez-Fornells A, Marco-Pallarés J (2012) The role of 
beta-gamma oscillations in unexpected rewards processing. Neuroimage 60:1678-1685. CrossRef Medline

Jasper H, Penfield W (1949) Electrocorticograms in man: effect of voluntary movement upon the electrical activity of the precentral gyrus. Arch Psychiatr Z Neurol 183:163-174. CrossRef

Jenkinson N, Brown P (2011) New insights into the relationship between dopamine, beta oscillations and motor function. Trends Neurosci 34: 611-618. CrossRef Medline

Kilavik BE, Zaepffel M, Brovelli A, MacKay WA, Riehle A (2013) The ups and downs of beta oscillations in sensorimotor cortex. Exp Neurol 245: 15-26. CrossRef Medline

Koelewijn T, van Schie HT, Bekkering H, Oostenveld R, Jensen O (2008) Motor-cortical beta oscillations are modulated by correctness of observed action. Neuroimage 40:767-775. CrossRef Medline

Korenberg A, Ghahramani Z (2002) A Bayesian view of motor adaptation. Curr Psychol Cogn 21:537-564.

Linkenkaer-Hansen K, Nikulin VV, Palva S, Ilmoniemi RJ, Palva JM (2004) Prestimulus oscillations enhance psychophysical performance in humans. J Neurosci 24:10186-10190. CrossRef Medline

Luft CD (2014) Learning from feedback: the neural mechanisms of feedback processing facilitating better performance. Behav Brain Res 261: 356-368. CrossRef Medline

Luft CD, Nolte G, Bhattacharya J (2013) High-learners present larger midfrontal theta power and connectivity in response to incorrect performance feedback. J Neurosci 33:2029-2038. CrossRef Medline

Luft CD, Takase E, Bhattacharya J (2014) Processing graded feedback: electrophysiological correlates of learning from small and large errors. J Cogn Neurosci 26:1180-1193. CrossRef Medline

Marco-Pallares J, Cucurell D, Cunillera T, García R, Andrés-Pueyo A, Münte TF, Rodríguez-Fornells A (2008) Human oscillatory activity associated to reward processing in a gambling task. Neuropsychologia 46:241-248. CrossRef Medline

Marco-Pallarés J, Münte TF, Rodríguez-Fornells A (2015) The role of highfrequency oscillatory activity in reward processing and learning. Neurosci Biobehav Rev 49:1-7. CrossRef Medline

Marko MK, Haith AM, Harran MD, Shadmehr R (2012) Sensitivity to prediction error in reach adaptation. J Neurophysiol 108:1752-1763. CrossRef Medline

Molnár M, Csuhaj R, Gaál ZA, Czigler B, Ulbert I, Boha R, Kondákor I (2008) Spectral characteristics and linear-nonlinear synchronization changes of different EEG frequency bands during the CNV. Psychophysiology 45: 412-419. CrossRef Medline

Müller GR, Neuper C, Rupp R, Keinrath C, Gerner HJ, Pfurtscheller G (2003) Event-related beta EEG changes during wrist movements induced by functional electrical stimulation of forearm muscles in man. Neurosci Lett 340:143-147. CrossRef Medline

Nagamine T, Kajola M, Salmelin R, Shibasaki H, Hari R (1996) Movementrelated slow cortical magnetic fields and changes of spontaneous MEG- and EEG-brain rhythms. Electroencephalogr Clin Neurophysiol 99:274-286. CrossRef Medline

Oldfield RC (1971) The assessment and analysis of handedness: the Edinburgh inventory. Neuropsychologia 9:97-113. CrossRef Medline

Paradiso G, Cunic D, Saint-Cyr JA, Hoque T, Lozano AM, Lang AE, Chen R (2004) Involvement of human thalamus in the preparation of self-paced movement. Brain 127:2717-2731. CrossRef Medline

Pfurtscheller G, Lopes da Silva FH (1999) Event-related EEG/MEG synchronization and desynchronization: basic principles. Clin Neurophysiol 110:1842-1857. CrossRef Medline

Pfurtscheller G, Stancák A Jr, Neuper C (1996) Post-movement beta synchronization. A correlate of an idling motor area? Electroencephalogr Clin Neurophysiol 98:281-293. CrossRef Medline

Prablanc C, Martin O (1992) Automatic control during hand reaching at undetected 2-dimensional target displacements. J Neurophysiol 67:455469. Medline

Ridderinkhof KR, Ullsperger M, Crone EA, Nieuwenhuis S (2004) The role of the medial frontal cortex in cognitive control. Science 306:443-447. CrossRef Medline
Roelfsema PR, Engel AK, König P, Singer W (1997) Visuomotor integration is associated with zero time-lag synchronization among cortical areas. Nature 385:157-161. CrossRef Medline

Saleh M, Reimer J, Penn R, Ojakangas CL, Hatsopoulos NG (2010) Fast and slow oscillations in human primary motor cortex predict oncoming behaviorally relevant cues. Neuron 65:461-471. CrossRef Medline

Siegel M, Donner TH, Engel AK (2012) Spectral fingerprints of largescale neuronal interactions. Nat Rev Neurosci 13:121-134. CrossRef Medline

Sober SJ, Brainard MS (2012) Vocal learning is constrained by the statistics of sensorimotor experience. Proc Natl Acad Sci U S A 109:21099-21103. CrossRef Medline

Takahashi K, Saleh M, Penn RD, Hatsopoulos NG (2011) Propagating waves in human motor cortex. Front Hum Neurosci 5:40. CrossRef Medline

Tamura Y, Hoshiyama M, Nakata H, Hiroe N, Inui K, Kaneoke Y, Inoue K, Kakigi R (2005) Functional relationship between human rolandic oscillations and motor cortical excitability: an MEG study. Eur J Neurosci 21:2555-2562. CrossRef Medline

Tan H, Jenkinson N, Brown P (2014a) Dynamic neural correlates of motor error monitoring and adaptation during trial-to-trial learning. J Neurosci 34:5678-5688. CrossRef Medline

Tan H, Zavala B, Pogosyan A, Ashkan K, Zrinzo L, Foltynie T, Limousin P, Brown P (2014b) Human subthalamic nucleus in movement error detection and its evaluation during visuomotor adaptation. J Neurosci 34: 16744-16754. CrossRef Medline

Taniguchi M, Kato A, Fujita N, Hirata M, Tanaka H, Kihara T, Ninomiya H, Hirabuki N, Nakamura H, Robinson SE, Cheyne D, Yoshimine T (2000) Movement-related desynchronization of the cerebral cortex studied with spatially filtered magnetoencephalography. Neuroimage 12:298-306. CrossRef Medline

Thoroughman KA, Shadmehr R (2000) Learning of action through adaptive combination of motor primitives. Nature 407:742-747. CrossRef Medline

Torrecillos F, Albouy P, Brochier T, Malfait N (2014) Does the processing of sensory and reward-prediction errors involve common neural resources? Evidence from a frontocentral negative potential modulated by movement execution errors. J Neurosci 34:4845-4856. CrossRef Medline

Tseng YW, Diedrichsen J, Krakauer JW, Shadmehr R, Bastian AJ (2007) Sensory prediction errors drive cerebellum-dependent adaptation of reaching. J Neurophysiol 98:54-62. CrossRef Medline

van Beers RJ (2009) Motor learning is optimally tuned to the properties of motor noise. Neuron 63:406-417. CrossRef Medline

van de Vijver I, Ridderinkhof KR, Cohen MX (2011) Frontal oscillatory dynamics predict feedback learning and action adjustment. J Cogn Neurosci 23:4106-4121. CrossRef Medline

van Ede F, Jensen O, Maris E (2010) Tactile expectation modulates prestimulus $\beta$-band oscillations in human sensorimotor cortex. Neuroimage 51:867-876. CrossRef Medline

van Ede F, de Lange F, Jensen O, Maris E (2011) Orienting attention to an upcoming tactile event involves a spatially and temporally specific modulation of sensorimotor alpha- and beta-band oscillations. J Neurosci 31:2016-2024. CrossRef Medline

van Wijk BCM, Daffertshofer A, Roach N, Praamstra P (2009) A role of beta oscillatory synchrony in biasing response competition? Cereb Cortex 19: 1294-1302. CrossRef Medline

Wei K, Körding K (2009) Relevance of error: what drives motor adaptation? J Neurophysiol 101:655-664. CrossRef Medline

Zaepffel M, Trachel R, Kilavik BE, Brochier T (2013) Modulations of EEG beta power during planning and execution of grasping movements. PLoS One 8:e60060. CrossRef Medline

Zhang Y, Wang X, Bressler SL, Chen Y, Ding M (2008) Prestimulus cortical activity is correlated with speed of visuomotor processing. J Cogn Neurosci 20:1915-1925. CrossRef Medline

Zohary E, Shadlen MN, Newsome WT (1994) Correlated neuronal discharge rate and its implications for psychophysical performance. Nature 370:140-143. CrossRef Medline 